\title{
Bedforms in a turbulent stream: ripples, chevrons and antidunes
}

\author{
By BRUNO ANDREOTTI, PHILIPPE CLAUDIN, \\ OLIVIER DEVAUCHELLE', ORENCIO DURÁN \\ AND ANTOINE FOURRIËRE \\ Laboratoire de Physique et Mécanique des Milieux Hétérogènes, \\ (PMMH UMR 7636 ESPCI - CNRS - Univ. Paris Diderot - Univ. P. M. Curie), \\ 10 rue Vauquelin, 75231, Paris Cedex 05, France. \\ ${ }^{\dagger}$ Department of Earth, Atmospheric and Planetary Sciences, Massachusetts Institute of \\ Technology, 77 Massachusetts Avenue, Cambridge MA 02139-4307, USA
}

(Received 15 September 2011)

The interaction between a turbulent flow and a granular bed via sediment transport produces various bedforms associated to distinct hydrodynamical regimes. In this paper, we compare ripples (downstream propagating transverse bedforms), chevrons and bars (bedforms inclined with respect to the flow direction) and anti-dunes (upstream propagating bedforms), focusing on the mechanisms involved in the early stages of their formation. Performing the linear stability analysis of a flat bed, we study the asymptotic behaviours of the dispersion relation with respect to the physical parameters of the problem. In the subcritical regime (Froude number $\mathcal{F}$ smaller than unity), we show that the same instability produces ripples or chevrons depending on the influence of the free surface. The transition from transverse to inclined bedforms is controled by the ratio of the saturation length $L_{\text {sat }}$, which encodes the stabilising effect of sediment transport, to the flow depth $H$, which determines the hydrodynamical regime. These results suggest that alternate bars form in rivers during flooding events, when suspended load dominates over bed load. In the supercritical regime $\mathcal{F}>1$, the transition from ripples to anti-dunes is also controlled by the ratio $L_{\text {sat }} / H$. Anti-dunes appear around resonant conditions for free surface waves, a situation for which the sediment transport saturation becomes destabilising. This resonance turns out to be fundamentally different from the inviscid prediction. Their wavelength selected by linear instability mostly scales on the flow depth $H$, which is in agreement with existing experimental data. Our results also predict the emergence, at large Froude numbers, of 'anti-chevrons' or 'anti-bars', i.e. bedforms inclined with respect to the flow and propagating upstream.

\section{Introduction}

Alluvial rivers often develop bedforms, which result from the unstable interaction between bed, sediment transport and water flow. The velocity field is perturbed by the presence of bedforms. In turn, the fluid motion induces sediment transport which, through erosion and deposition, deforms the bed (Julien 1998). Depending on the flow depth and velocity, as well as on the sediment properties, various patterns can be observed (Ashley 1990). Ripples and dunes migrate downstream, and their crest is orthogonal to the flow direction (Fig. 1a). The wavelength $\lambda$ of ripples is much smaller than the water depth $H$, so that the flow around these bedforms is not in- 

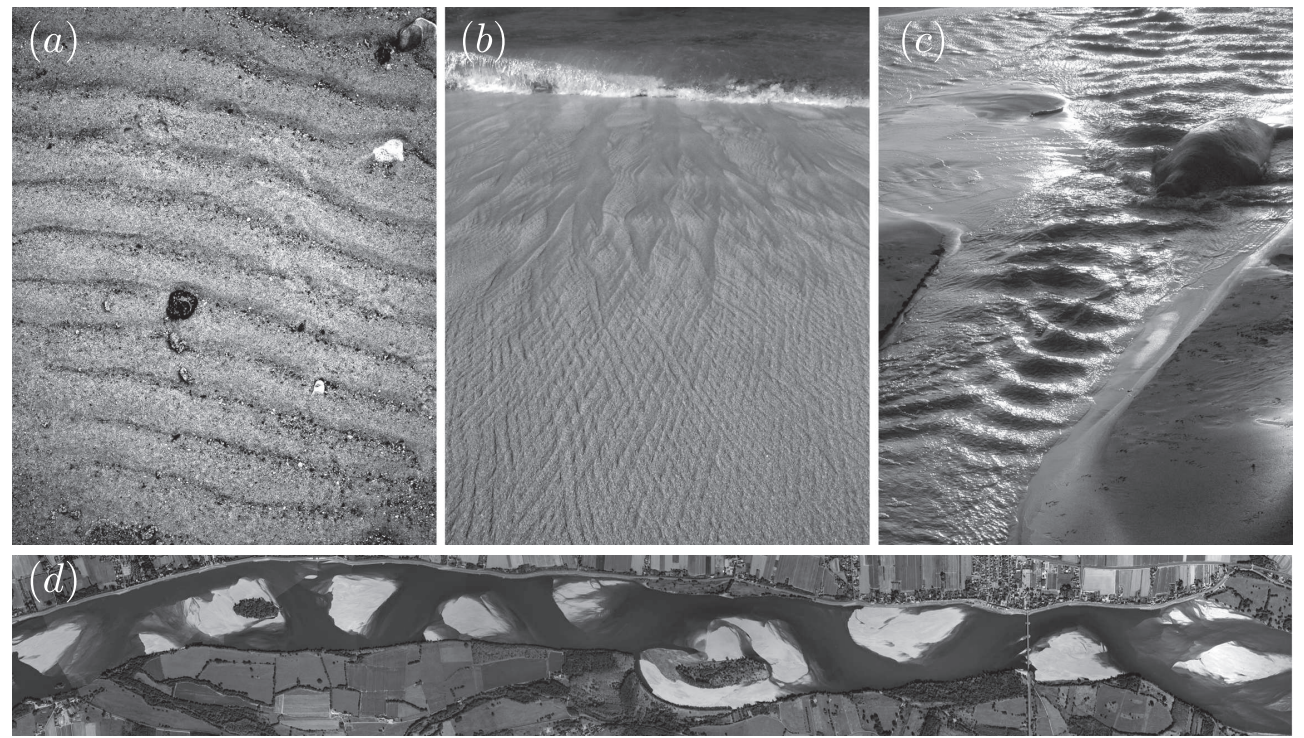

Figure 1. (a) Subaqueous ripples (Zion National Park, USA, $37^{\circ} 16^{\prime} \mathrm{N} 112^{\circ} 57^{\prime} \mathrm{W}$ ); photo credit: B. Andreotti. The crests are transverse to the flow (from top to bottom). (b) Chevrons formed by backswash on the beach (Honokai Hale, Hawaii, $21^{\circ} 21^{\prime} \mathrm{N} 158^{\circ} 08^{\prime} \mathrm{W}$ ); photo credit: L. Langevin. (c) Antidunes (California, USA, $35^{\circ} 41^{\prime} \mathrm{N} 121^{\circ} 17^{\prime} \mathrm{W}$ ); photo credit: B. Andreotti. The sea lion gives the typical scale. (d) Alternate bars in the Loire river (France, $47^{\circ} 24^{\prime} \mathrm{N} 0^{\circ} 22^{\prime} \mathrm{W}$ ); photo credit: Digital Globe.

fluenced by the water surface. By contrast, dunes have a typical length comparable to, or larger than $H$, and thus interact with the free surface. Although dunes and ripples were previously seen as two distinct modes of the same linear instability (Richards 1980, Sumer \& Bakioglu 1984, McLean 1990), it is now proposed that dunes result from the coarsening of ripples, through the non-linear increase of their wavelength (Raudkivi \& Witte 1990, Raudkivi 2006, Fourrière et al. 2010). Such a coarsening has been reported in numerous experimental studies (Mantz 1978, Gyr \& Schmid 1989, Baas 1994,

Coleman \& Melville 1994a, Baas 1999, Robert \& Uhlman 2001, Coleman et al. 2003, Venditti et al. 2005b, Langlois \& Valance 2007, Rauen et al. 2008).

Unlike dunes and ripples, the crest of what is called 'chevrons' or 'rhomboid pattern' (Fig. 1b) forms an angle $\alpha$ with the flow direction (Woodford 1935, Chang \& Simons 1970, Morton 1978, Karcz \& Kersey 1980, Ikeda 1983, Daerr et al. 2003, Devauchelle et al. 2010a). Chevrons migrate downstream and interact with the free surface. Alternate bars in rivers and channels can be thought of as the superimposition of two rhomboid patterns with opposite angles (Fig. 1d). The boundary conditions at the bank select their transverse wavenumber, the channel acting as a wave guide for the chevrons instability. Several stability analyses have been devoted to these bedforms, related to meandering and braiding in rivers (e.g., Callander 1969, Parker 1976, Fredsøe 1978, Tubino et al. 1999). Flume experiments have also been performed to reproduce alternate bars at the laboratory scale (e.g. Chang et al. 1971, Schumm \& Khan 1972, Ikeda 1983, Fujita \& Muramoto 1985, Lisle et al. 1991, Lisle et al. 1997, Lanzoni 2000a, Lanzoni 2000b). Interestingly, these bars are observed when the submergence, i.e. the ratio between the water depth $H$ and the grain size $d$, is moderate and the Froude number $\mathcal{F}$ is below unity (the Froude number compares inertia with gravity). Some figures of Fujita \& Muramoto 1985 suggest that alternate bars result from a linear instability, but Lanzoni 2000a reports the emergence 
of ripples first. Unfortunately, few contributions describe the early stages of the bed evolution.

Antidunes form in supercritical flows, that is for a Froude number larger than unity, and migrate upstream (Fig. 1c). They have received much experimental and theoretical attention (e.g. Raudkivi 1966, Ikeda 1983, Alexander et al. 2001, Carling \& Shvidchenko 2002), either on their own (Parker 1975, Kubo \& Yokokawa 2001, Colombini \& Stocchino 2005) or in association with other bedforms (Kennedy 1963, Reynolds 1965, Engelund 1970, Hayashi 1970, Huang \& Chiang 2001, Colombini 2004, Colombini \& Stocchino 2008). Steppool sequences (Chin 1999, Curran 2007, Lenzi 2001, Weichert, et al., 2008, Whittaker and Jaeggi, 1982) and cyclic steps (Parker \& Izumi 2000, Kostic et al. 2010) are extreme forms of antidunes, the growth of which has caused the flow to cross periodically the transition from subcritical to supercritical regimes.

The primary linear instability causing ripples and chevrons results from the phase lag between sediment flux $q$ and bed topography $Z$ (Kennedy 1963, Hayashi 1970, Parker 1975, Richards 1980, Engelund \& Fredsøe 1982, McLean 1990). This phase lag has two main contributions (Andreotti et al. 2002). First, there is a hydrodynamical effect. For a wavelength $\lambda$ sufficiently small with respect to the water depth $H$ (ripples), fluid inertia causes a phase advance of the basal shear stress $\tau$, which is destabilising. However, when $\lambda$ compares with $H$, the confinement of the flow can cause a phase delay of $\tau$ with respect to $Z$. The second contribution to the phase lag between $Z$ and $q$ is related to the relaxation of the sediment flux towards equilibrium. This stabilizing effect involves a length scale $L_{\text {sat }}$, the so-called saturation length. Its value depends on the mode of sediment transport. For bedload it is about ten times the grain diameter $d$ in a turbulent flow (Fourrière et al. 2010). For suspended transport, the saturation length scales with the water depth (Claudin et al. 2011). It corresponds to the distance a grain travels horizontally before settling down.

In sections 2 and 3, we generalize the stability analysis of Fourrière et al. (2010) to non-transverse patterns, in order to investigate the emergence of chevrons and bars, and we determine the growth rate $\sigma$ associated to a bedform of wavenumber $k=2 \pi / \lambda$ and of angle $\alpha$. Section 4 is devoted to the analysis of the problem in the limit of a vanishing Froude number $(\mathcal{F} \rightarrow 0)$. We show that the ratio between the flow depth $H$ and the saturation length $L_{\text {sat }}$ controls the transition from transverse patterns (ripples) to inclined patterns (chevrons and bars). Finally, section 5 is devoted to finite Froude numbers effects and in particular to the transition from ripples to antidunes.

\section{Sediment transport}

In order to predict the emergence of bedforms from a flat sedimentary bed, one needs to model erosion and deposition of particles. The aim of this section is to show that the different modes of sediment transport can be described within the very same theoretical framework. This has already been discussed in a series of papers investigating the relaxation of sediment transport towards equilibrium (Parker 1975, Sauermann et al. 2001, Andreotti et al. 2002, Charru 2006, Andreotti \& Claudin 2007, Andreotti et al. 2010, Fourrière et al. 2010, Claudin et al. 2011). We propose here a summary of these results.

\subsection{Transport regimes}

Figure 2 shows that the two modes of underwater sediment transport, namely bedload and suspended load, can be distinguished by the profile of the grain concentration $\phi$ in the mobile phase. Neglecting inertia, the particle velocity equals that of the fluid. In turbulent flows, the water velocity fluctuations tend to homogenise the sediment concen- 

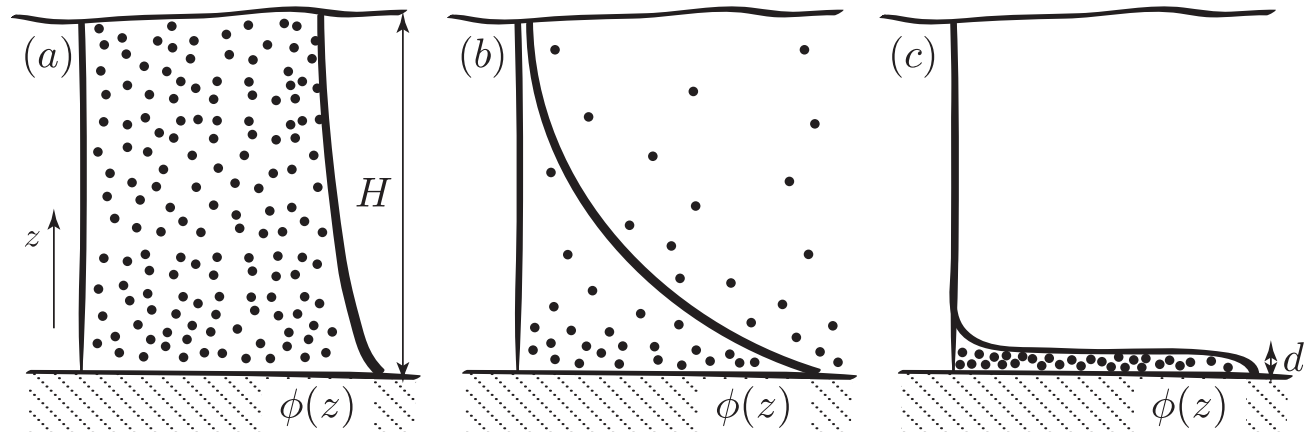

FiguRE 2. Sediment concentration profile $\phi(z)$ for different sedimentation velocities $V_{\text {fall }}$. (a) At small sedimentation velocity, the particles are suspended by turbulent fluctuations and occupy the entire flow. (b) Transitional case. (c) Conversely, at high sedimentation velocity, the sediment flux is concentrated near the bed, in a transport layer whose thickness is proportional to $d$.

tration $\phi$. This effect is balanced by gravity, which tends to settle the particles down at a velocity $V_{\text {fall }}$. In the low concentration limit, the sedimentation flux is therefore $\varphi_{\downarrow}=V_{\text {fall }} \phi$. The simplest model taking these two effects into account is:

$$
\partial_{t} \phi+\vec{u} \cdot \vec{\nabla} \phi=\vec{\nabla} \cdot(D \vec{\nabla} \phi)+V_{\text {fall }} \partial_{z} \phi
$$

where $D$ is an effective turbulent diffusion coefficient and $\vec{u}$ the velocity field. For the sake of simplicity, we consider here $D$ as a constant proportional to the mean flow velocity $U$ (or equivalently, to the shear velocity $u_{*}$ ) and to the flow thickness $H$, which controls the typical turbulent mixing length: $D=\beta H U$. In the homogeneous steady state called the saturated state in this context - the upward diffusive flux is balanced by the downward sedimentation flux:

$$
D \partial_{z} \phi=-V_{\text {fall }} \phi .
$$

Introducing the basal concentration $\phi_{b}$, this equation integrates into

$$
\phi=\phi_{b} \exp \left(-\frac{V_{\text {fall }}}{\beta U} \frac{z}{H}\right)
$$

This expression provides a good approximation to experimental data (Rouse, 1936; Vanoni, 1946; van Rijn, 1984b). The dimensionless parameter $V_{\text {fall }} / U$ controls the transition between suspended transport and bed load. At small sedimentation velocity, turbulent fluctuations are more efficient than gravity so that the concentration profile is homogeneous (Fig. 2a). Sediment transport takes place over the entire flow thickness $H$. Conversely, at large sedimentation velocity, gravity concentrates the moving particles at the surface of the bed (Fig. 2c). In this limit, the sediment transport layer is limited by the grain diameter $d$.

\subsection{Turbulent suspension}

We now consider more specifically the asymptotic regime $V_{\text {fall }} / U \ll 1$, which corresponds to turbulent suspension. The basal concentration and thus the overall transport are governed by the rate of entrainment $\varphi_{\uparrow}$ of grains from the static sand bed (or 'pick-up function'). As this entrainment results from the hydrodynamical drag on the grains, $\varphi_{\uparrow}$ is controlled by the basal shear stress $\tau_{x z}$ or equivalently by the shear velocity $u_{*} \equiv$ $\sqrt{\tau_{x z} / \rho_{f}}$. Empirical measurements show that $\varphi_{\uparrow}$ is a growing function of $u_{*}$ above a threshold $u_{\text {th }} \propto V_{\text {fall }}$ (van Rijn, 1984a), typically proportional to $\left(u_{*}^{2}-u_{\mathrm{th}}^{2}\right)$ (Partheniades, 
1965). The deposition rate $\varphi_{\downarrow}$ is due to gravity, which tends to trap the moving grains. The conservation of sediment mass then gives the time evolution of the bed profile $Z:$ :

$$
\partial_{t} Z=\varphi_{\downarrow}-\varphi_{\uparrow}
$$

Neglecting the volume of particles in suspension, this equation can be formulated in terms of the sediment volume flux $q=\int_{0}^{H} \phi u d z$ as:

$$
\partial_{t} Z+\partial_{x} q=0
$$

The value of $q$ in the steady homogeneous state is called the saturated flux.

In the saturated regime, the concentration $\phi$ is almost homogeneous and equal to $\phi_{b}$, which is selected by the mass conservation across the interface between static and mobile grains: $\varphi_{\downarrow}=V_{\text {fall }} \phi_{b}=\varphi_{\uparrow}\left(u_{*}\right)$. One gets the scaling

$$
\phi \simeq \phi_{b} \propto \frac{u_{*}^{2}-u_{\mathrm{th}}^{2}}{V_{\text {fall }}}
$$

The saturated flux thus reads

$$
q_{\mathrm{sat}} \simeq \phi_{b} U H=\frac{\varphi_{\uparrow}\left(u_{*}\right) U H}{V_{\text {fall }}} \propto \frac{H u_{*}\left(u_{*}^{2}-u_{\mathrm{th}}^{2}\right)}{V_{\text {fall }}}
$$

By assumption, the suspended load takes place far from the threshold, so that $q_{\text {sat }}$ scales as $u_{*}^{3}$.

We now consider the transient of saturation of suspended transport in a heterogeneous situation, following the analysis proposed by Claudin et al. 2011. We consider a sinusoidal perturbation of the basal shear stress along $x$ at a small wavenumber $(k H \ll 1)$, which leads to a perturbation $\varphi_{\uparrow}^{1}$ of the sediment flux. Bedforms with short wavelength $(k H \gg 1)$ are ignored here as they are quickly erased in the suspended regime. Then, horizontal diffusion of particles can be neglected. In the steady state, the diffusion equation (2.1) reduces to:

$$
U \partial_{x} \phi=\partial_{z}\left(\beta U H \partial_{z} \phi+V_{\text {fall }} \phi\right)
$$

The perturbation $\phi_{1}$ to the concentration field at saturation reads

$$
\phi_{1}=\left[F_{+} \exp \left(K_{+} z / H\right)+F_{-} \exp \left(K_{-} z / H\right)\right] e^{i k x}
$$

where, at leading order in $V_{\text {fall }} / U$ and $k H$, the dimensionless parameters $K_{+}$and $K_{-}$ are given by

$$
K_{ \pm} \sim-\frac{V_{\text {fall }}}{2 \beta U} \pm(1+i) \sqrt{\frac{k H}{2 \beta}}
$$

The constant $F_{+}$and $F_{-}$are fixed by the two boundary conditions: (i) the particle flux must vanish at the free surface $(z=H$ ); (ii) the diffusive flux is equal to the erosion flux $\varphi_{\uparrow}^{1}$ at the bed surface $(z=0)$.

Using equations (2.4) and (2.5), the variations of the flux can be related to erosion and deposition rates: $\partial_{x} q=\varphi_{\uparrow}-\varphi_{\downarrow}$. This leads to a relation between the flux perturbation $q_{1}$ and the entrainment rate perturbation $\varphi_{\uparrow}^{1}$. After a short calculation, one obtains, in the limit of small $V_{\text {fall }} / U$ and $k H$, the expression

$$
\varphi_{\uparrow}^{1} \sim\left(i k+\frac{V_{\text {fall }}}{U H}\right) q_{1},
$$

which does not depend anymore on $\beta$. Using equation (2.7), one can compute the saturated flux perturbation $q_{\text {sat }}^{1}=\varphi_{\uparrow}^{1} U H / V_{\text {fall }}$, associated to the modulation of the basal 
shear stress. One observes that the actual sediment flux is not equal to the saturated flux:

$$
q_{1}=\frac{q_{\mathrm{sat}}^{1}}{1+i \frac{U}{V_{\text {fall }}} k H}
$$

This equation corresponds to a first order space relaxation of the form

$$
L_{\mathrm{sat}} \partial_{x} q=q_{\mathrm{sat}}-q,
$$

where the relaxation length, called the saturation length in this context, reads

$$
L_{\text {sat }} \simeq \frac{U}{V_{\text {fall }}} H .
$$

The physical interpretation of this relation is as follows. The sediment flux adapts to variations of the basal shear stress with a space lag $L_{\mathrm{sat}}$. In other words, there is a space delay between erosion, controlled by the basal shear stress, and sediment flux, which is due to the length needed to mix particles. The time needed for a particle to settle down over the flow thickness $H$ is $H / V_{\text {fall }}$. Multiplying by the horizontal velocity $U$, one obtains the distance a particle travels before settling. Typically, the saturation length for suspended transport $L_{\text {sat }}$ ranges from $10 H$ to $100 H$ (Claudin et al. 2011). This calculation can be easily adapted to the two-dimensional situation. The relaxation equation keeps the same form (2.13) except that the flux is now a vector (see Eq. (2.20) below). Even in this case, the relaxation only takes place along the flow direction $x$.

The most important conclusion of this section is that erosion and deposition are controlled the basal shear stress. Turbulent mixing in the bulk only causes a space lag which can be abstracted into a single length-scale: the saturation length $L_{\text {sat }}$.

\subsection{Bedload}

We now consider the opposite asymptotic regime which corresponds to bed load transport. In this case, turbulent fluctuations are too weak to homogenize the particle concentration. Charru 2006 and Lajeunesse et al. 2010 have proposed that turbulent bedload obey the same physics as turbulent suspension, except that the particles are transported in a layer of characteristic thickness the grain diameter $d$, instead of $H$. According to this point of view, the saturated sediment flux reads

$$
q_{\mathrm{sat}} \simeq \phi_{b} U d=\frac{\varphi_{\uparrow}\left(u_{*}\right) U d}{V_{\text {fall }}} \propto \frac{d u_{*}\left(u_{*}^{2}-u_{\mathrm{th}}^{2}\right)}{V_{\text {fall }}}
$$

The transient towards saturation in the case of bedload transport can be described by the same relaxation equation (2.13) as suspended load except that the saturation length now scales as:

$$
L_{\text {sat }} \propto \frac{U}{V_{\text {fall }}} d
$$

As for the suspended regime, this is the length needed for a grain to settle down across a layer of thickness $d$.

A different line of thought originates from Bagnold's description of sediment transport, which is based on momentum conservation (Bagnold 1956). Contrarily to the previous picture, grains are transported with a dominant horizontal velocity. Then, the difference between the total shear stress $\rho_{f} u_{*}^{2}$ applied to the transport layer, and the residual shear stress $\rho_{f} u_{\mathrm{th}}^{2}$ at the surface of the static layer is supported by the deeper layers of the bed. The difference $\rho_{f}\left(u_{*}^{2}-u_{\mathrm{th}}^{2}\right)$, called the sand-borne shear stress, is the number $n$ of grains transported per unit surface times the friction force exerted by the static bed on 
the mobile grains. One thus deduces the scaling law followed by $n$,

$$
n \propto \frac{1}{d^{2}}\left(\frac{u_{*}^{2}}{u_{\mathrm{th}}^{2}}-1\right)
$$

This scaling law was confirmed experimentally by Lajeunesse et al. 2010. Multiplying $n$ by the grain velocity, which increases linearly with $u_{*}$, one recovers a saturated flux scaling as $u_{*}^{3}$ for large $u_{*}$ (Meyer-Peter \& Müller 1948, Einstein 1950, Fernandez Luque \& van Beek 1976, Lajeunesse et al. 2010). In this picture, the length needed for transport to adjust to a change of basal shear stress results from inertia. It is simply the length needed by a grain to reach its equilibrium horizontal velocity. As the fluid drag force is proportional to the fluid density $\rho_{f}$ while the mass of the grains is proportional to the grain density $\rho_{p}$, one obtains a saturation length scaling as:

$$
L_{\text {sat }} \propto \frac{\rho_{p}}{\rho_{f}} d
$$

It has been shown by Fourrière et al. 2010 that $L_{\text {sat }}$ can be determined experimentally from initial ripple wavelength. Further work is needed to discriminate between the two dynamical mechanisms (deposition length vs inertial length) that can control the saturation length. The key test would be to investigate systematically the influence of the density ratio $\rho_{p} / \rho_{f}$ on the initial ripples wavelength. In both cases, the saturation length is on the order of few grain diameters and is, by contrast, much smaller in the bedload than in the suspended load regime.

\subsection{Framework for the general description of sediment transport}

In the two previous paragraphs, we have seen that the different modes of sediment transport lead to different saturated fluxes and saturation lengths, but can be described in the same formalism. We have detailled above the case of bed-load and suspended load in the turbulent regime but aeolian transport in saltation (Andreotti et al. 2002, Andreotti et al. 2010) or bed-load in the viscous regime (Charru 2006) are also consistent with the saturation length framework. We thus emphasise that the type of sediment transport does not change the physics of bedform dynamics.

Let us generalise the elements of this descriptive framework at three dimensions. We define the volumic flux $\vec{q}$, which is a two components vector $\left(q_{x}, q_{y}\right)$, from the conservation of sediment mass:

$$
\partial_{t} Z+\vec{\nabla} \cdot \vec{q}=0
$$

The saturated flux $\vec{q}_{\text {sat }}$ is by definition the flux in the homogeneous steady state. It is a function of the basal shear stress $\vec{\tau}=\tau \vec{t}$, which is also a two components vector. The sediment flux relaxes towards its saturated value $\vec{q}_{\text {sat }}=q_{\text {sat }} \vec{t}$ over a typical distance $L_{\text {sat }}$ along the direction of the flow:

$$
L_{\mathrm{sat}}(\vec{t} \cdot \vec{\nabla}) \vec{q}=\vec{q}_{\mathrm{sat}}-\vec{q}
$$

The relaxation equation (2.20) presents two interesting limits. When $L_{\text {sat }}$ tends to 0 , the flux is always saturated and is thus a function of the shear stress. When $L_{\text {sat }}$ tends to infinity, it is the erosion rate $\partial_{t} Z$ which is controlled by the shear stress.

For the sake of generality, we write the saturated flux on a flat sand bed as

$$
\vec{q}_{\mathrm{sat}}=\Omega\left(u_{*}^{2}-u_{\mathrm{th}}^{2}\right)^{\gamma} \vec{t},
$$

where $\Omega$ is a dimensionfull constant of proportionality and $\gamma$ is an exponent (as previously seen, $\gamma \simeq 3 / 2$ for bed-load and suspension). For simplicity, we first neglect the influence 


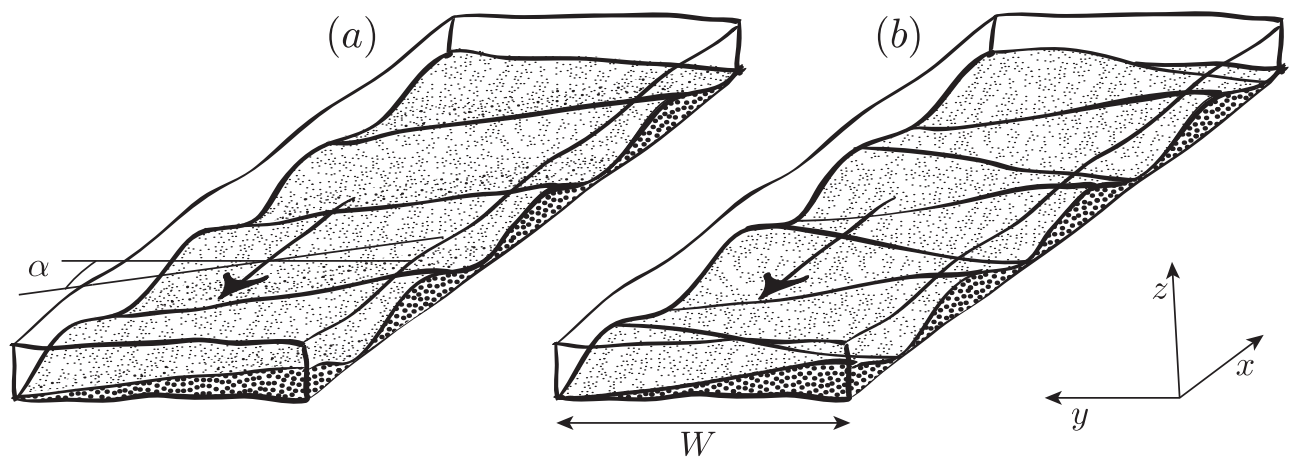

Figure 3. Schematic of bedforms at an angle $\alpha$ with respect to the flow. (a) Plane wave. (b) Alternate bars (guided waves in a channel of width $W$ ). The $x$-axis is in the direction of the flow (black arrows), and $y$ is transverse to it. $z$ is the third coordinate, perpendicular to the bed mean plane. We note $Z(x, y)$ the bed profile.

of the bed slope on both the threshold shear velocity $u_{\mathrm{th}}$ and the direction of the saturated flux, postponing the discussion of this effect to section 4.5. We have checked that the results presented in this article are robust in the sense that they are not qualitatively affected by the precise choice of such a transport law.

\section{Hydrodynamics}

\subsection{Dispersion relation}

To predict the emergence of bedforms, the purpose of hydrodynamics is to determine the shear stress exerted by the fluid on the bed, since the later controls sediment transport. The periodic disturbance of the bed profile $Z$ is treated as a perturbation of a base state that is homogeneous. We can then seek for modes of the form

$$
Z=\zeta e^{i k(\cos \alpha x+\sin \alpha y-c t)+\sigma t}
$$

where $\alpha$ is the angle between the wavevector $\vec{k}=(k \cos \alpha, k \sin \alpha)$ and the flow direction (Fig. 3), $\sigma$ is the growth rate and $c$ is the propagation velocity of the pattern. For a linear stability analysis, one needs to perform the linear expansion of the flow equations with respect to the small parameter $k \zeta$. Denoting by a superscript $\wedge$ the space Fourier transform, the basal stress $\tau_{i j}$ can be related to the elevation profile $Z$ by

$$
\hat{\tau}_{x z}=-u_{*}^{2}\left(A_{x}+i B_{x}\right) k \hat{Z}, \quad \text { and } \quad \hat{\tau}_{y z}=-u_{*}^{2}\left(A_{y}+i B_{y}\right) k \hat{Z}
$$

$A_{x}, B_{x}, A_{y}$ and $B_{y}$ are respectively the components of the shear stress in phase $\left(A_{x}\right.$ and $\left.A_{y}\right)$ and in quadrature with the bed deformation $\left(B_{x}\right.$ and $\left.B_{y}\right)$. Due to the scale separation between the typical evolution time of the bedforms and that of the flow, the hydrodynamical velocity field can be considered in a steady state at each time. As a consequence, these coefficients are independent of $\sigma$ and $c$ (Fourrière et al. 2010). The aim of a particular hydrodynamical description is to relate these four dimensionless coefficients to the wavenumber $k$ and the angle $\alpha$. We will propose such a technical derivation in section 3.3 and focus first on the qualitative aspects of hydrodynamics above bedforms.

For given $A_{x}, B_{x}, A_{y}$ and $B_{y}$, one can compute the dispersion relation for bedforms, using the saturation length formalism. The flux relaxation towards its saturated value 


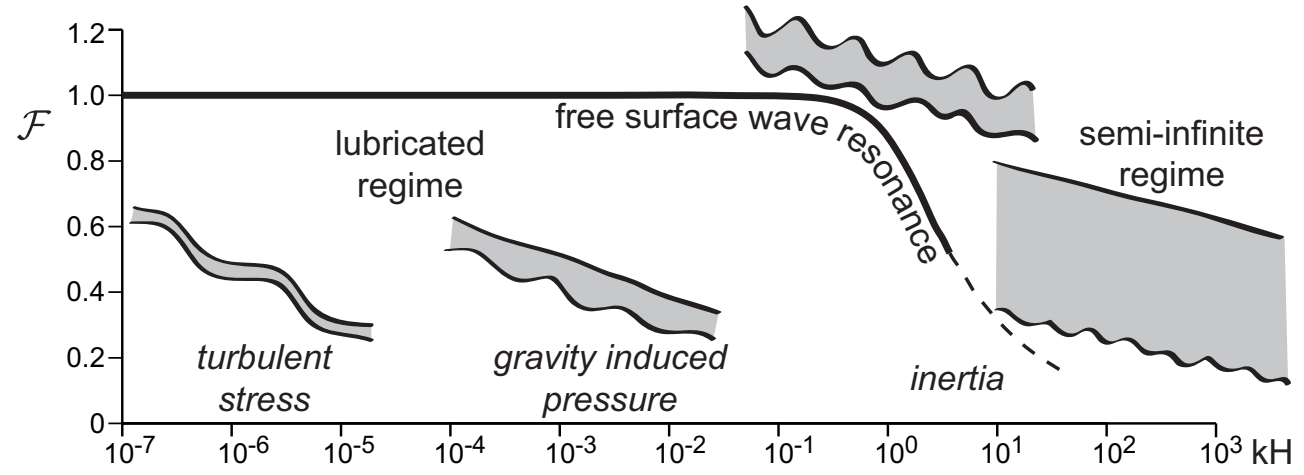

Figure 4. Diagram of the hydrodynamic regimes as a function of the Froude number $\mathcal{F}$ and the wavelength normalized by the flow thickness, $k H$. Black thick line: resonance curve predicted by the inviscid theory. Grey schematics: qualitative shape of the free surface with respect to the undulations of the bottom. Roman text: hydrodynamical regimes. Italic text: relevant mechanism dominating hydrodynamics.

takes the form:

$$
i k \cos \alpha L_{\mathrm{sat}} \hat{q}_{x}=\hat{q}_{\mathrm{sat}}^{x}-\hat{q}_{x} \quad \text { and } \quad i k \cos \alpha L_{\mathrm{sat}} \hat{q}_{y}=\hat{q}_{\mathrm{sat}}^{y}-\hat{q}_{y} .
$$

Defining the reference flux $Q=\gamma \Omega\left(u_{*}^{2}-u_{\mathrm{th}}^{2}\right)^{\gamma-1} u_{*}^{2}$, the saturated flux can be related to the shear stresses $\tau_{x z}$ and $\tau_{y z}$ induced by the wavy bed as:

$$
\frac{\hat{q}_{\mathrm{sat}}^{x}}{Q}=-\frac{\hat{\tau}_{x z}}{u_{*}^{2}} \quad \text { and } \quad \frac{\hat{q}_{\mathrm{sat}}^{y}}{Q}=-\frac{1}{\gamma}\left(1-\frac{u_{\mathrm{th}}^{2}}{u_{*}^{2}}\right) \frac{\hat{\tau}_{y z}}{u_{*}^{2}} .
$$

Finally the conservation equation leads to:

$$
\sigma-i k c=-i k \cos \alpha \frac{\hat{q}_{x}}{\hat{Z}}-i k \sin \alpha \frac{\hat{q}_{y}}{\hat{Z}} .
$$

One then obtains the following dispersion relation:

$$
\sigma-i k c=-\frac{i k^{2} Q}{1+i k \cos \alpha L_{\mathrm{sat}}}\left(\cos \alpha\left(a_{x}+i b_{x}\right)+\sin \alpha\left(a_{y}+i b_{y}\right)\right),
$$

where $a_{x}=A_{x}, b_{x}=B_{x}, a_{y}=\left(1-\frac{u_{\mathrm{th}}^{2}}{u_{*}^{2}}\right) A_{y} / \gamma$ and $b_{y}=\left(1-\frac{u_{\mathrm{th}}^{2}}{u_{*}^{2}}\right) B_{y} / \gamma$ are the components of the saturated flux in and out of phase with the topography. There are two interesting limits that will be considered below in details. When the shear stress is much larger that the threshold value (i.e. for infinite $u_{*} / u_{\text {th }}$ ) one gets $a_{x}=A_{x}, b_{x}=B_{x}$, $a_{y}=A_{y} / \gamma$ and $b_{y}=B_{y} / \gamma$. Conversely, just above the threshold, one gets instead $a_{x}=A_{x}, b_{x}=B_{x}, a_{y}=0$ and $b_{y}=0$. As a consequence, the transverse component of the flux is negligible in this limit.

\subsection{Hydrodynamical regimes}

One can identify four hydrodynamical regimes (Fig. 4), in which these functions $A$ and $B$ have different behaviours. In the limit of short wavelength $(k H \gg 1$, semi-infinite regime), the turbulent flow over a wavy bed can be decomposed into three regions:

- Outer layer - In the outer layer, away from the bottom, the pressure gradient is mostly balanced by inertia; turbulent stresses are sub-dominant. The streamlines follow the topography so that the velocity at the bottom of the outer layer is in phase with the bottom. 
- Inner layer - In the inner layer, the inertial terms of the Navier-Stokes equation are sub-dominant, and the longitudinal pressure gradient is thus balanced by the Reynolds shear stress transverse gradient, i.e. by the mixing of momentum due to turbulent fluctuations (Fourrière et al. 2010). The phase lag between basal shear stress and topography results from the matching of the inner and outer layers in the region where inertial terms become comparable to stress gradients (Jackson \& Hunt 1975, Hunt et al. 1988). This balance relates the thickness $\ell$ of the inner layer to wavelength through $\lambda \sim \ell \ln ^{2}\left(\ell / z_{0}\right)$, where $z_{0}$ is the roughness length. At the transition between inner and outer layers, the fluid velocity is slowed down by the shear stress. In the limit of a small aspect ratio $k \zeta$, the velocity, which is inherited from the outer layer, is always phase delayed with respect to the shear stress, due to inertia. As a consequence, the shear stress is phase advanced with respect to the topography (positive $B$ coefficients), which means that the shear stress reaches its maximum upstream of the bedform crest. The phase lags of the shear stress $B_{x} / A_{x}$ and $B_{y} / A_{y}$ vanish for asymptotically small $k z_{0}$ and increase as $\ln \left(k z_{0}\right)$ for a larger wavenumber.

- Surface layer - The surface layer, of thickness $h_{0}$, is responsible for the hydrodynamical roughness $z_{0}$ seen from the inner layer. The dominant physical mechanism at work in this layer can be of different nature. For instance, $z_{0}$ can result from the mixing due to roughness elements, the predominance of viscous dissipation, or the presence of bed-load transport. The shear stress profile is insensitive to the mechanisms at work in the surface layer, provided that its thickness $h_{0}$ is smaller than the inner layer thickness $\ell$ : the hydrodynamical roughness $z_{0}$ is then the sole quantity inherited from the surface layer.

When the inner layer invades the whole system, the basal shear stress becomes sensitive to the free surface waves induced by the bedforms. This occurs when $\ell \simeq H$, or equivalently when the wavelength $\lambda$ is of the order of, or larger than $H \ln ^{2}\left(H / z_{0}\right)$. When the standing waves resonate with the bedforms - when the upstream propagation speed of surface waves at this wavelength is exactly balanced by the downstream convective velocity - the free surface is strongly deformed and the shear stress is phase delayed with respect to the bedforms (negative $B$ coefficients). For a wavelength larger than the resonant one, the confinement of the flow by the free surface becomes dominant. Three dynamical mechanisms balance: inertia, bed friction - which results from turbulent stress - and gravity-induced pressure. Contrary to the case of a deep flow, these three mechanisms are important over the entire flow depth. Their relative importance determines the regimes of figure 4 . We refer the interested reader to the OSM for a detailed hydrodynamical analysis of these various cases. When the gravity-induced pressure dominates the dynamics, the free surface is almost flat. By contrast, when the turbulent stress is dominant, the free surface follows the bed topography.

\subsection{Linear expansion}

Neither the potential flow approximation nor the Saint-Venant equations can satisfactorily describe hydrodynamics above bedforms: the first ignores turbulent shear stress and the second cannot account for the vertical structure of the flow (the layers we just described). We use here the same Reynolds averaged description of turbulence over a wavy bed as in Fourrière et al. (2010) (see references therein). We have checked that the results obtained are robust with respect to the details of the turbulent closure (anisotropy, lag between the shear rate and the resulting turbulent stress, surface layer description). We note $u_{i}$ the fluid velocity. Introducing the strain rate tensor $\dot{\gamma}_{i j}=\partial_{i} u_{j}+\partial_{j} u_{i}$ and its 
squared modulus $|\dot{\gamma}|^{2}=\frac{1}{2} \dot{\gamma}_{i j} \dot{\gamma}_{i j}$, the Reynolds stress $\tau_{i j}$ is expressed in a tensorial form:

$$
\tau_{i j}=\kappa^{2} L^{2}|\dot{\gamma}|\left(\frac{1}{3} \chi^{2}|\dot{\gamma}| \delta_{i j}-\dot{\gamma}_{i j}\right)
$$

where the density $\rho$ is set to unity. $\kappa \simeq 0.4$ is the von Kármán constant. $\chi$ is a second phenomenological constant in the range $2.5-3$. Considering a river inclined at an angle $\theta$ with respect to the horizontal plane, the shear stress $\tau_{x z}$ must balance gravity. It thus varies linearly as $\tau_{x z}=g(z-H) \sin \theta$ and vanishes at the free surface. By definition of the shear velocity $u_{*}$, we also write $\tau_{x z} \equiv u_{*}^{2}(z / H-1)$. The mixing length is chosen equal to $L=\left(z+z_{0}\right) \sqrt{1-z / H}$, a choice which results in a logarithmic flow profile for the base state, in accordance with field and experimental observations:

$$
u_{x}=\frac{u_{*}}{\kappa} \ln \left(1+\frac{z}{z_{0}}\right) .
$$

The average velocity $U$ then reads

$$
U=\frac{u_{*}}{\kappa}\left(1+\frac{z_{0}}{H}\right) \ln \left(1+\frac{H}{z_{0}}\right)-1 .
$$

The stress balance equation along the $z$-axis sets the pressure field:

$$
p+\tau_{z z}=p_{0}+g(H-z) \cos \theta=p_{0}+\frac{u_{*}^{2}}{\tan \theta}\left(1-\frac{z}{H}\right) .
$$

We define the surface Froude number $\mathcal{F}$ as the ratio of the surface water velocity to the velocity of gravity surface waves (in the shallow water limit):

$$
\mathcal{F} \equiv \frac{u_{x}(z=H)}{\sqrt{g H}}=\frac{1}{\kappa} \ln \left(1+\frac{H}{z_{0}}\right) \sqrt{\sin \theta} .
$$

The Froude number can be of order unity in flumes but is generally small for large natural rivers, due to their small slopes.

We now perform the formal derivation of the hydrodynamical equations. We consider the wavy bottom defined by Eq. 3.1 and we perform the linear expansion of the equations with respect to the small parameter $k \zeta$. We note $\eta=k z, \eta_{0}=k z_{0}$ and $\eta_{H}=k H$. We write the first order corrections to the base flow as

$$
\begin{aligned}
u_{x} & =u_{*}[\mu+k Z U], \\
u_{y} & =u_{*} k Z V \\
u_{z} & =u_{*} k Z W \\
\tau_{x z} & =\tau_{z x}=-u_{*}^{2}\left[1-\frac{\eta}{\eta_{H}}+k Z S_{x z}\right], \\
\tau_{y z} & =\tau_{z y}=-u_{*}^{2}\left[k Z S_{y z}\right] \\
\tau_{x y} & =\tau_{y x}=-u_{*}^{2}\left[k Z S_{x y}\right], \\
p+\tau_{z z} & =p_{0}+u_{*}^{2}\left[\frac{1}{\tan \theta}\left(1-\frac{\eta}{\eta_{H}}\right)+k Z S_{n}\right], \\
\tau_{x x} & =u_{*}^{2}\left[\frac{1}{3} \chi^{2}+k Z S_{x x}\right] \\
\tau_{y y} & =u_{*}^{2}\left[\frac{1}{3} \chi^{2}+k Z S_{y y}\right],
\end{aligned}
$$




$$
\tau_{z z}=u_{*}^{2}\left[\frac{1}{3} \chi^{2}+k Z S_{z z}\right]
$$

where the function $\mu$ is defined by $\mu(\eta)=\frac{1}{\kappa} \ln \left(\frac{\eta}{\eta_{0}}\right)$. The capitalized quantities $U, V$, $W, S_{x z}, S_{y z}$, and $S_{n}$ are functions of $\eta$. The free surface is also deformed and we denote by $h=H+\Delta(x, y)$ the flow depth at the position $x, y$. The modified expression for the mixing length then reads

$$
L=\left(z_{0}+z-Z\right) \sqrt{\frac{H+\Delta-z}{H+\Delta-Z}} .
$$

At first order, the stress equations can be simplified into

$$
\begin{aligned}
& \mu^{\prime} S_{x z}=2\left(1-\frac{\eta}{\eta_{H}}\right)\left(\left(U^{\prime}+i \cos \alpha W\right)-\kappa \mu^{\prime 2}+\frac{\mu^{\prime}}{2 \eta_{H}}+\frac{\eta \delta \mu^{\prime}}{2 \eta_{H}^{2}\left(1-\frac{\eta}{\eta_{H}}\right)}\right) \\
& \mu^{\prime} S_{x y}=\left(1-\frac{\eta}{\eta_{H}}\right)(i \sin \alpha U+i \cos \alpha V) \\
& \mu^{\prime} S_{y z}=\left(1-\frac{\eta}{\eta_{H}}\right)\left(V^{\prime}+i \sin \alpha W\right) \\
& \mu^{\prime}\left(S_{x x}-S_{z z}\right)=\left(1-\frac{\eta}{\eta_{H}}\right)\left(-2 i \cos \alpha U+2 W^{\prime}\right) \\
& \mu^{\prime}\left(S_{y y}-S_{z z}\right)=\left(1-\frac{\eta}{\eta_{H}}\right)\left(-2 i \sin \alpha V+2 W^{\prime}\right)
\end{aligned}
$$

The Navier-Stokes equations lead to

$$
\begin{aligned}
W^{\prime} & =-i \cos \alpha U-i \sin \alpha V \\
S_{x z}^{\prime} & =i \mu \cos \alpha U+\mu^{\prime} W+i \cos \alpha\left(S_{n}+S_{x x}-S_{z z}\right)-i \sin \alpha S_{x y} \\
S_{y z}^{\prime} & =i \mu \cos \alpha V+i \sin \alpha\left(S_{n}+S_{y y}-S_{z z}\right)-i \cos \alpha S_{y x} \\
S_{n}^{\prime} & =-i \mu \cos \alpha W+i \cos \alpha S_{x z}+i \sin \alpha S_{y z} .
\end{aligned}
$$

Introducing the vector $\vec{X}=\left(U, V, W, S_{x z}, S_{y z}, S_{n}\right)$, at first order in $k \zeta$, one has to solve a system of six differential equations which can be written as

$$
\frac{d}{d \eta} \vec{X}=\mathcal{P} \vec{X}+\vec{S}+\delta \overrightarrow{S_{\delta}}
$$

where

$$
\mathcal{P}=\left(\begin{array}{cccccc}
0 & 0 & -i \cos \alpha & \frac{\mu^{\prime}}{2\left(1-\frac{\eta}{\eta_{H}}\right)} & 0 & 0 \\
0 & 0 & -i \sin \alpha & 0 & \frac{\mu^{\prime}}{\left(1-\frac{\eta}{\eta_{H}}\right)} & 0 \\
-i \cos \alpha & -i \sin \alpha & 0 & 0 & 0 \\
\frac{1+3 \cos ^{2} \alpha}{\mu^{\prime}}\left(1-\frac{\eta}{\eta_{H}}\right)+i \mu \cos \alpha & \frac{3 \sin \alpha \cos \alpha}{\mu^{\prime}}\left(1-\frac{\eta}{\eta_{H}}\right) & \mu^{\prime} & 0 & 0 & i \cos \alpha \\
\frac{3 \sin \alpha \cos \alpha}{\mu^{\prime}}\left(1-\frac{\eta}{\eta_{H}}\right) & \frac{1+3 \sin ^{2} \alpha}{\mu^{\prime}}\left(1-\frac{\eta}{\eta_{H}}\right)+i \mu \cos \alpha & 0 & 0 & 0 & i \sin \alpha \\
0 & 0 & -i \mu \cos \alpha & i \cos \alpha & i \sin \alpha & 0
\end{array}\right),
$$




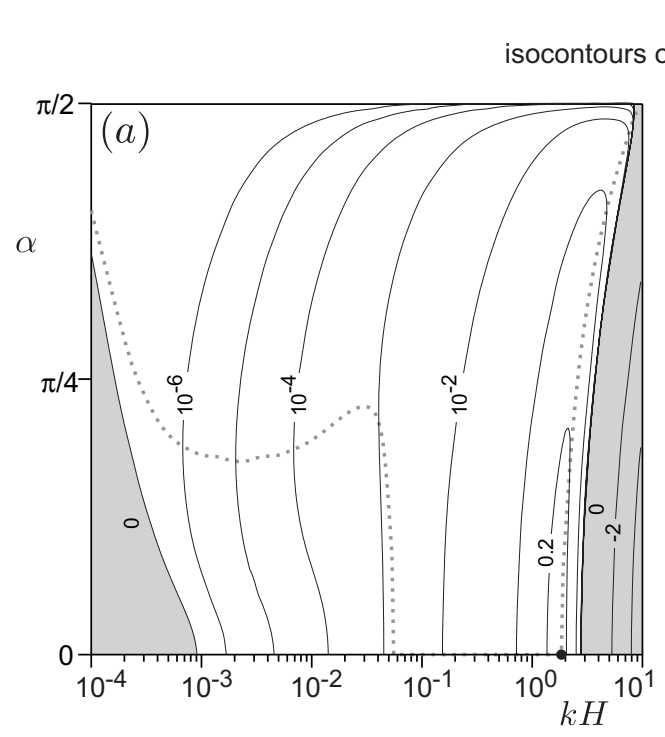

$\frac{L_{\text {sat }}^{2} \sigma}{Q}$

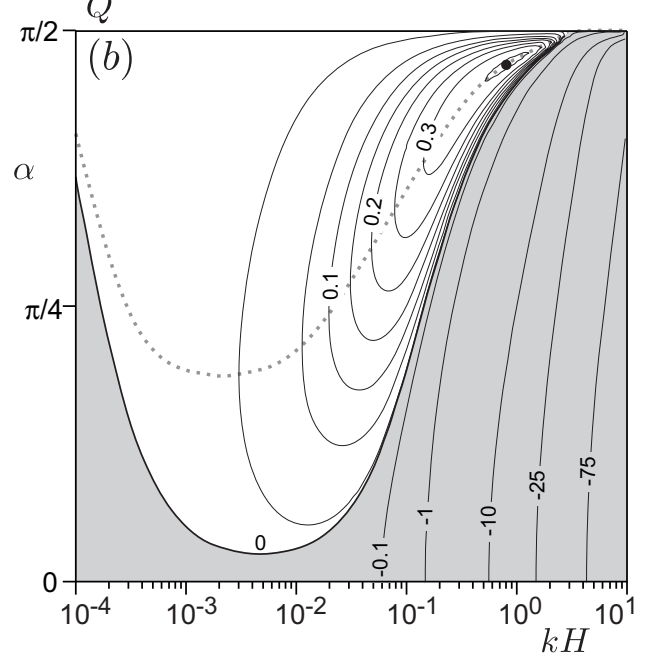

FiguRE 5. Growth rate $\sigma$ as a function of the rescaled wavenumber $k H$ and of the angle $\alpha$ for $u_{*} / u_{\text {th }} \rightarrow \infty$ and $\mathcal{F} \rightarrow 0$ (a) Data computed for $L_{\mathrm{sat}} / H=1 / 4$. The most unstable mode $(\bullet)$ corresponds to ripples $(\alpha=0)$. Isocontours for $L_{\mathrm{sat}}^{2} \sigma / Q=-5,-2,0,10^{-6}, 10^{-5}, 10^{-4}, 10^{-3}, 10^{-2}, 10^{-1}, 0.2$. (b) Data for $L_{\mathrm{sat}} / H=4$. The influence of the free surface on the basal shear stress drives the most unstable mode towards higher $\alpha$ values. Isocontours for $L_{\text {sat }}^{2} \sigma / Q=-75,-25,-10,-1,-0.1,0,0.005,0.05,0.1,0.15,0.2,0.25,0.3,0.35$. The dotted lines correspond to the maximum over all possible values of $\alpha$ of growth rate, for a given $k H$.

$\vec{S}=\left(\begin{array}{c}\kappa \mu^{\prime 2}-\frac{\mu^{\prime}}{2 \eta_{H}} \\ 0 \\ 0 \\ 0 \\ 0\end{array}\right)$, and $\quad \overrightarrow{S_{\delta}}=\left(\begin{array}{c}-\frac{\eta \mu^{\prime}}{2 \eta_{H}^{2}\left(1-\frac{\eta}{\eta_{H}}\right)} \\ 0 \\ 0 \\ 0 \\ 0 \\ 0\end{array}\right)$.

The bottom boundary conditions $U(0)=-1 /\left(\kappa \eta_{0}\right), V(0)=0$ and $W(0)=0$ are then automatically satisfied - as the flow above the bedform is assumed to be in a steady state, the water velocity must vanish at the bed surface. At the free surface, the normal velocity vanishes $W\left(\eta_{H}\right)=i \mu\left(\eta_{H}\right) \cos \alpha \delta$, as well as the stress $S_{x z}\left(\eta_{H}\right)=\delta / \eta_{H}, S_{y z}\left(\eta_{H}\right)=0$ and $S_{n}\left(\eta_{H}\right)=\delta /\left(\eta_{H} \tan \theta\right)$. These conditions select the coefficients $S_{x z}(0)=A_{x}+i B_{x}$, $S_{y z}(0)=A_{y}+i B_{y}, S_{n}(0)$ and $\delta$. Integrating equation (3.34) yields to the dependence of these stress coefficients on $k$ and $\alpha$.

\section{Transition from transverse to inclined bedforms}

In this section, the dispersion relation derived above is first analysed in its simplest version, that is for a vanishing Froude number and a sediment transport model independent of the bed slope. Then, we will introduce the influence of the bed slope in subsection 4.5 and investigate the influence of the Froude number in section 5. 

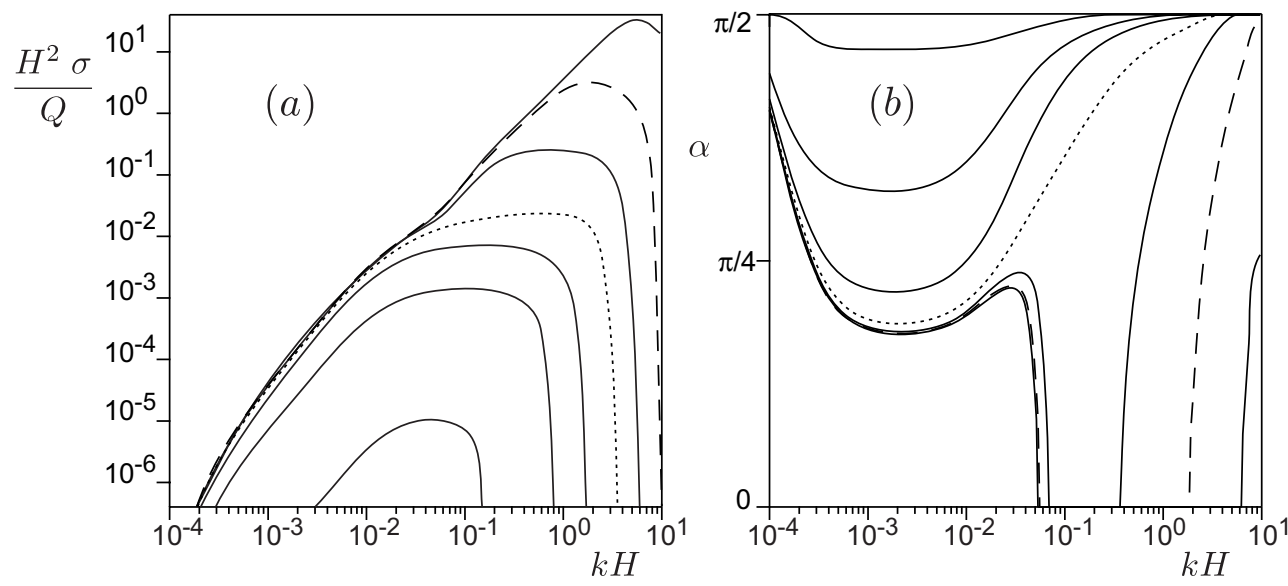

FiguRE 6. Maximum growth rate (a) and corresponding mode angle (b) computed over all possible $\alpha$, for each wavenumber $k H$. Curves obtained in the limit $\mathcal{F} \rightarrow 0, u_{*} / u_{\mathrm{th}} \rightarrow \infty$, and for $L_{\text {sat }} / H=2^{-4}, 2^{-2}, 2^{0}, 2^{2}, 2^{4}, 2^{6}, 2^{8}$. The dashed line corresponds to $L_{\text {sat }} / H=1 / 4$ (Fig. 5a) and the dotted line to $L_{\mathrm{sat}} / H=4$ (Fig. $5 \mathrm{~b}$ ).

\subsection{Non-dimensional parameters}

To produce a phase diagram of the possible bedform patterns, the different non-dimensional parameters of the model must be identified. Choosing $H$ as a characteristic length scale to rescale the wavenumber $k$, there remain two other lengths in the problem: the hydrodynamical roughness $z_{0}$ and the saturation length $L_{\text {sat }}$. The relative influence of inertia and gravity on hydrodynamics is characterized by the Froude number $\mathcal{F}$. The relative influence of fluid entrainment and gravity on sediment transport is characterized by the ratio $u_{*} / u_{\mathrm{th}}$. The linear stability analysis of a flat sand bed in a river thus depends on four parameters, $H / z_{0}, L_{\mathrm{sat}} / H, \mathcal{F}$ and $u_{*} / u_{\mathrm{th}}$, which can be varied independently in experiments, at least in principle, by changing the bed material. There are two further non-dimensional numbers in the transport law: its exponent $\gamma$ and the effective friction coefficient $\mu$ of the granular material. As the central result of this article, we show that the most important parameter is the ratio $L_{\text {sat }} / H$, since it controls the transition from ripples to chevrons. More precisely, $L_{\text {sat }}$ controls the stabilizing effect associated to transport and $H$ the hydrodynamical regime. The other parameters do not affect the nature and the physical origin of this transition even though they have a quantitative influence on the results, especially in the neighbourhood of this transition.

This central result determines the strategy adopted here to investigate the dependence of the instability on six parameters. We first consider the low Froude number limit, that is when inertial effects at the free surface are negligible with respect to gravity. For illustrative purposes, the ratio $H / z_{0}$ is set to 100 , which is realistic for rivers. Similarly, $\gamma$ is fixed to $3 / 2$ and $\mu^{-1}$ to 0 . Finally, the results will be systematically plotted for the two extreme values of $u_{*} / u_{\mathrm{th}}, 1$ and $+\infty$. So that, we are left with a single non-dimensional number $L_{\mathrm{sat}} / H$, which controls the instability.

\subsection{Growth rate in the low Froude number limit}

Figure 5 shows the iso-contours of the growth rate $\sigma$ as a function of the wavenumber rescaled by the flow thickness, $k H$, and of the pattern angle $\alpha$. Two values of $L_{\text {sat }} / H$ have been chosen, which are on both sides of the transition between ripples and chevrons. The growth rate shown in panel (a) of figure 5 is computed for a small value of $L_{\mathrm{sat}} / H=1 / 4$. Let us first focus on the horizontal axis $\alpha=0$, on which the maximum of the growth 

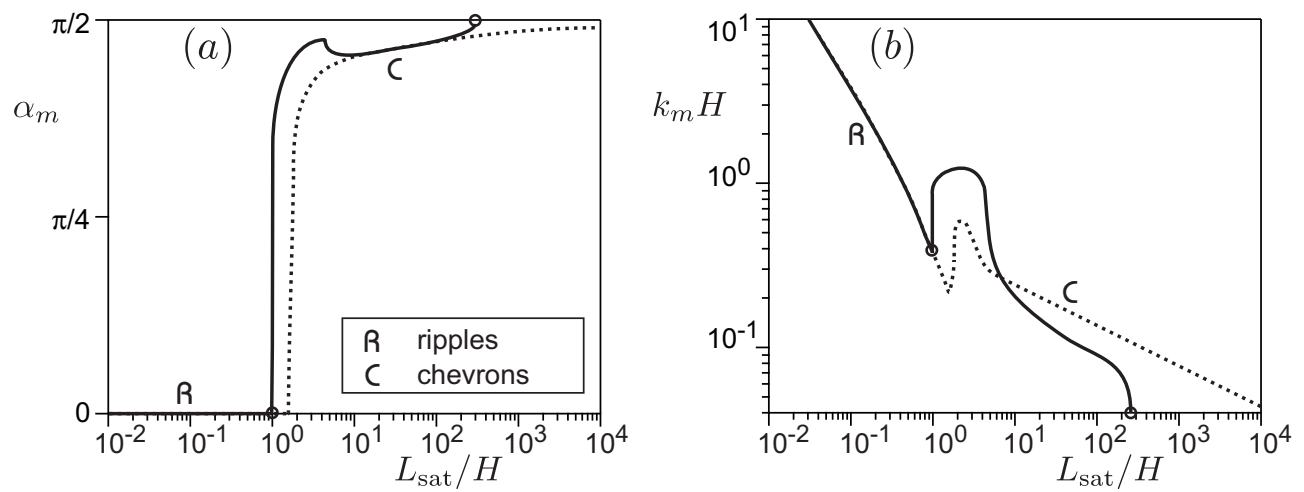

FiguRE 7. Angle $\alpha_{m}$ and wavenumber $k_{m}$ of the most unstable mode as a function of $L_{\text {sat }} / H$, for $\mathcal{F} \rightarrow 0$. The solid line corresponds to the limit $u_{*} / u_{\mathrm{th}} \rightarrow \infty$ (transitions at $L_{\text {sat }} / H \simeq 1.0$ and at $\simeq 2.910^{2}$ marked by $\circ$ ) and the dotted line to the limit $u_{*} / u_{\mathrm{th}} \rightarrow 1$ (transitions at $\left.L_{\text {sat }} / H \simeq 1.6\right)$.

rate is located, and for which one recovers the results of Fourrière et al. (2010). The region of negative growth rate for $k H \ll 1$ (gray zone on the left-hand side) is due to flow confinement. In this limit, the flow is controlled by the balance between gravity induced slope effect and turbulent friction on the bottom (Fig. 4). Therefore, the disturbance to the base state corresponds to a down-slope velocity component $\left(B_{x}<0\right)$. This results into a diffusive sediment flux which tends to stabilise the bedform $(\sigma<0)$. As the wavenumber increases, inertia becomes more and more important so that the phase delay of the shear stress with respect to the topography is progressively reduced. When the confinement effect becomes negligible, one recovers a phase advance $\left(B_{x}>0\right)$ and thus a destabilising hydrodynamical effect.

In the semi-infinite regime, when the wavelength is much larger than the saturation length $\left(k L_{\text {sat }} \gg 1\right)$, the growth rate is a growing function of $k H$. This results from mass conservation, as the amount of sediment to be transported is smaller for shorter wavelengths, while the flow-induced destabilising effect (the coefficient $B_{x}$ ) remains of the same intensity. The re-stabilisation at larger wavenumbers (gray zone on the right-hand side) is associated to the saturation length $L_{\text {sat }}$. The maximum growth rate is determined by the balance between the stabilising effect of transport and the destabilising effect of hydrodynamics. It is convenient to define the most unstable wavenumber $k_{\infty}$ in the limit $H \gg L_{\text {sat }}$, for a given ratio $L_{\text {sat }} / z_{0} . k_{\infty}$ is the product of $L_{\text {sat }}^{-1}$ by a dimensionless factor of hydrodynamical origin corresponding to the phase lag of the shear stress with respect to the topography.

Let us consider a given granular bed ( $L_{\text {sat }}$ is fixed) under different flow conditions. When $H$ is large enough, the free surface does not influence the flow, and the most unstable mode corresponds to ripples $(\alpha=0)$ of wavenumber $k_{\infty}$. When decreasing the flow depth $H$ to values comparable to, or smaller than $L_{\text {sat }}$, the influence of the free surface on the flow increases. The panel (b) of figure 5 corresponds to such a large value of the saturation length $\left(L_{\mathrm{sat}} / H=4\right)$. One observes that all transverse patterns $(\alpha=0)$ are stable $(\sigma<0)$. However, some inclined patterns, which correspond to chevrons, remain unstable.

Why is the confinement by the free surface stabilising for quasi-transverse patterns ( small $\alpha$ ) but destabilising for inclined patterns (large $\alpha$ )? Consider first the limit $k H \ll$ 1 for which the balance between slope effect and basal friction dominates. Due to gravity, for any value of $\alpha$, the disturbance to the flow is associated to a fluid motion that 


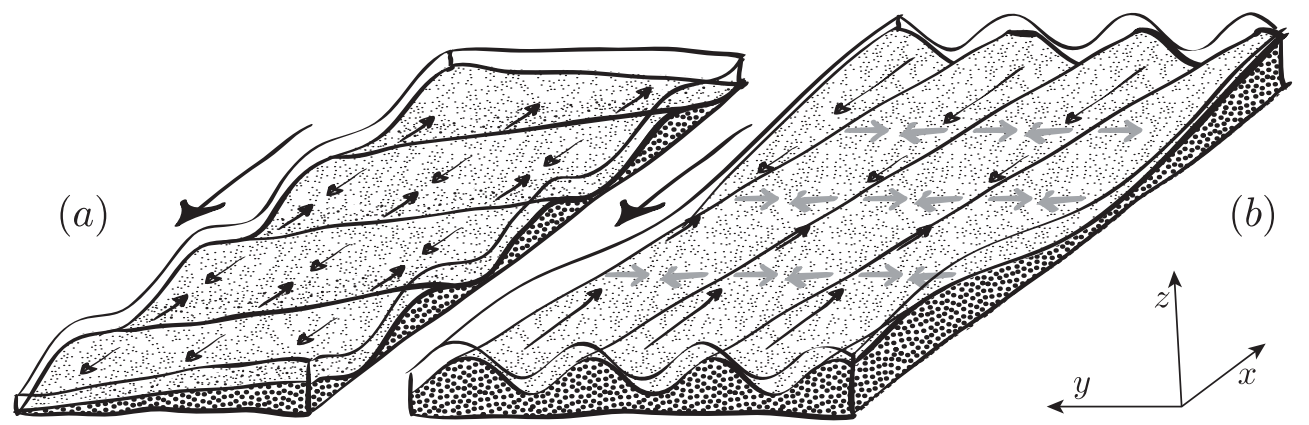

FIGURE 8. Schematic explanation of the chevrons instability mechanism in the limit of vanishing $k H$. Black (resp. grey) arrows show the modulation of the longitudinal (transverse) flow. (a) Bedforms almost transverse to the flow ( $\alpha$ close to 0). Gravity is dominant so that the longitudinal fluid motion is down-slope. (b) Bedforms almost aligned with the flow ( $\alpha$ close to $\pi / 2)$. The longitudinal slope effect is negligible. Longitudinal fluid motion is controlled by the modulation of flow thickness, which induces a weakening (resp. an increase) of the basal friction upstream (resp. downstream) of the crest. Therefore, the modulation of the longitudinal fluid motion is up-slope.

is globally down slope. For small $\alpha$, this corresponds to a stabilising down-slope flow $\left(B_{x}<0\right)$, as shown in Fig. 8(a). However, at $\alpha$ close to $\pi / 2$, the slope effect mostly induces a motion along the direction perpendicular to the main flow. The longitudinal slope effect is small and can be ignored. As there is no net water discharge from the crests to the trough, the fluid motion is down-slope along the transverse direction but must be up-slope along the longitudinal direction $\left(B_{x}>0\right)$. More precisely, as shown in Fig. 8(b), the transverse flow induces a modulation of the flow thickness in quadrature with topography: the flow thickness is larger up-stream of the bedform crest and smaller down-stream of it. In turn, turbulent friction is modulated and leads to the up-slope longitudinal flow modulation. Note that these up-slope and down-slope water discharges take place over the whole flow thickness, due to confinement. Sediment transport is mostly controlled by the longitudinal modulation of the shear stress. As a consequence, the very same dynamical mechanism (balance between friction and slope effect) leads to a stabilising ( $\operatorname{small} \alpha$ ) or a destabilising (large $\alpha$ ) effect of the free surface. For any $\alpha$, this effect becomes weaker as $k H$ increases, since inertia becomes more and more important.

Computing, for given values of $k H$ and $L_{\text {sat }} / H$, the maximum over all possible angles $\alpha$ of the growth rate (dotted lines in Fig. 5, and Fig. 6) allows us to represent the dispersion relation in a more convenient form than a contour plot. Figure 6 a shows this maximum growth rate for different values of $L_{\mathrm{sat}} / H$ as a function of $k H$. These curves present a maximum, which is lower when $L_{\mathrm{sat}} / H$ is increased. This can be understood as the sediment transport saturation is a stabilising mechanism. In figure $6 \mathrm{~b}$, we represent the corresponding angles. We can see that, for all wavenumbers smaller than $k H \approx 0.1$, the growth rate is larger at a finite angle than at $\alpha=0$. At larger values of $k H$, there is a range of wavenumbers for which the largest growth rate is at $\alpha=0$. However, this range exists only for a sufficiently small ratio $L_{\text {sat }} / H$, and is wider for smaller values of this parameter. We will see in the next section that the most unstable modes (the maximum of the curves in figure 6a) precisely belong to this range for which $\alpha=0$ when $L_{\text {sat }} / H$ is small (transverse ripples), whereas it corresponds to chevrons (finite $\alpha$ ) when $L_{\mathrm{sat}} / H$ is large. 
Bedforms in a turbulent stream:

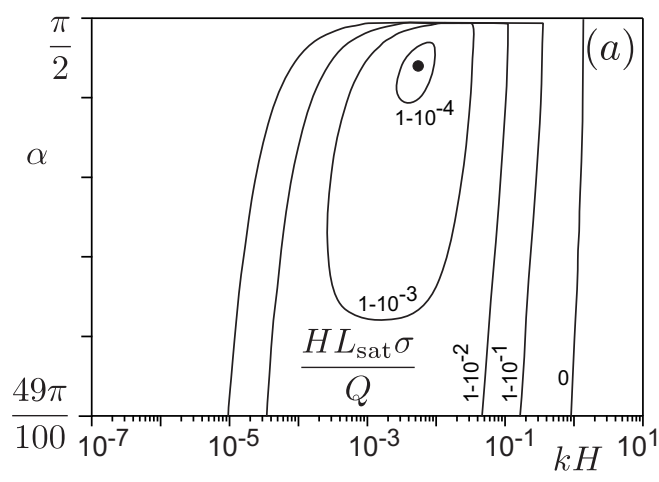

ripples, chevrons and antidunes

17

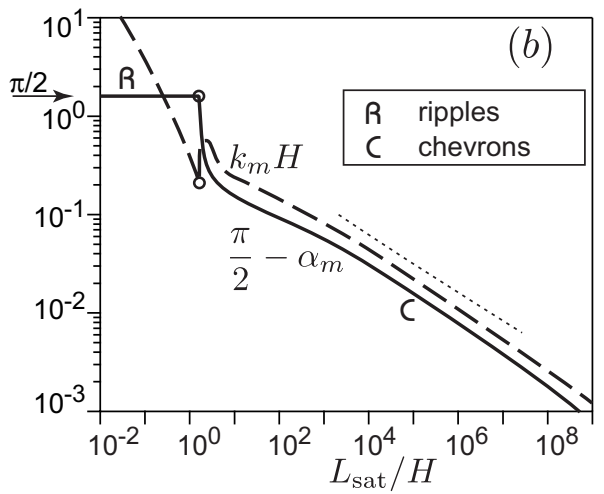

Figure 9. Erosive limit: incision instability. (a) Growth rate $\sigma$ as a function of the rescaled wavenumber $k H$ and of the angle $\alpha$ for $L_{\text {sat }} / H=10^{7}$ in the limit $\mathcal{F} \rightarrow 0$ and $u_{*} / u_{\mathrm{th}} \rightarrow 1$. The isocontours are taken close to 1 , for $L_{\text {sat }} H \sigma / Q-1=-10^{4},-10^{3},-10^{2},-10^{1},-10^{0}$. (b) Wavenumber $k_{m}$ (solid line) and angle $\pi / 2-\alpha_{m}$ (dashed line) of the most unstable mode as a function of $L_{\text {sat }} / H$, for $\mathcal{F} \rightarrow 0$. The dotted line is a guide for the eyes and is a power law of exponent -0.3 .

\subsection{Transition from ripples to chevrons}

From the dispersion relations shown in figure 6 , one can deduce the wavenumber $k_{m}$ and the angle $\alpha_{m}$ of the most unstable mode for which the growth rate is the largest (also shown in figure 5 by a black point). They are represented in figure 7 in the two limits $u_{*} / u_{\mathrm{th}} \rightarrow \infty$ and $u_{*} / u_{\mathrm{th}} \rightarrow 1$. The two cases behave very similarly. For both, there exist a threshold in $L_{\text {sat }} / H$, of order one, below which ripples are more unstable than chevrons. Below this transition at small $L_{\text {sat }} / H$, the ripples wavenumber $k_{m}$ is proportional to $L_{\text {sat }}^{-1}$ and thus does not depend on $H$. The behaviour beyond the transition is more complex, as it depends on both the flow depth $H$ and the saturation length $L_{\text {sat }}$.

There are small differences between the limits $u_{*} / u_{\mathrm{th}} \rightarrow 1$ and $u_{*} / u_{\mathrm{th}} \rightarrow \infty$, related to the effect (or the absence of effect) of the transverse shear stress. Close to the threshold, only the longitudinal shear stress matters. Although it is difficult to appreciate from figure 7 , due to the logarithmic scale, the transition from ripples to chevrons is much sharper far from the transport threshold (solid line) than close to it (dotted line). Moreover, the critical value of $L_{\mathrm{sat}} / H$ changes by a factor $\simeq 1.6$ between these two limits. More important is the difference at large ratio $L_{\text {sat }} / H$. In the limit $u_{*} / u_{\text {th }} \rightarrow \infty$, the entire spectrum of bedforms becomes stable beyond a critical value of the ratio $L_{\text {sat }} / H$ : a flat bed becomes stable toward any disturbance.

On the contrary, for $u_{*} / u_{\mathrm{th}} \rightarrow 1$, the angle $\alpha_{m}$ tends to $\pi / 2$ in the limit $L_{\text {sat }} / H \rightarrow \infty$. The most unstable mode in this limit thus corresponds to longitudinal structures. This can be interpreted as an incision instability, similar to that investigated by Izumi \& Parker 1995, 2000. Here however, we find that, far above the threshold, this longitudinal instability disappears (Fig. 7). Moreover, the incision wavelength is selected without any inhomogeneity in the base flow (Fig. 9). More precisely, figure 9 shows that the most unstable wavelength $k_{m}$ scales (roughly) as $k_{m} \propto H^{-0.7} L_{\text {sat }}^{-0.3}$. Similarly, the most unstable mode is inclined at an angle $\pi / 2-\alpha_{m} \propto\left(H / L_{\text {sat }}\right)^{0.3}$ with respect to the longitudinal direction. So, up to the limit of the numerical resolution, reached at $\sim L_{\text {sat }} / H=10^{9}, k_{m}$ still depends both on $H$ and $L_{\text {sat }}$ (in the whole range $0<\mathcal{F}<1$ ).

On the other hand, one could interpret the system in the limit $L_{\mathrm{sat}} / H \rightarrow \infty$ as a purely erosive material, with no deposition. The erosion rate is then controlled by the shear stress. Close to the threshold, the dispersion relation is then simply $\sigma-$ 

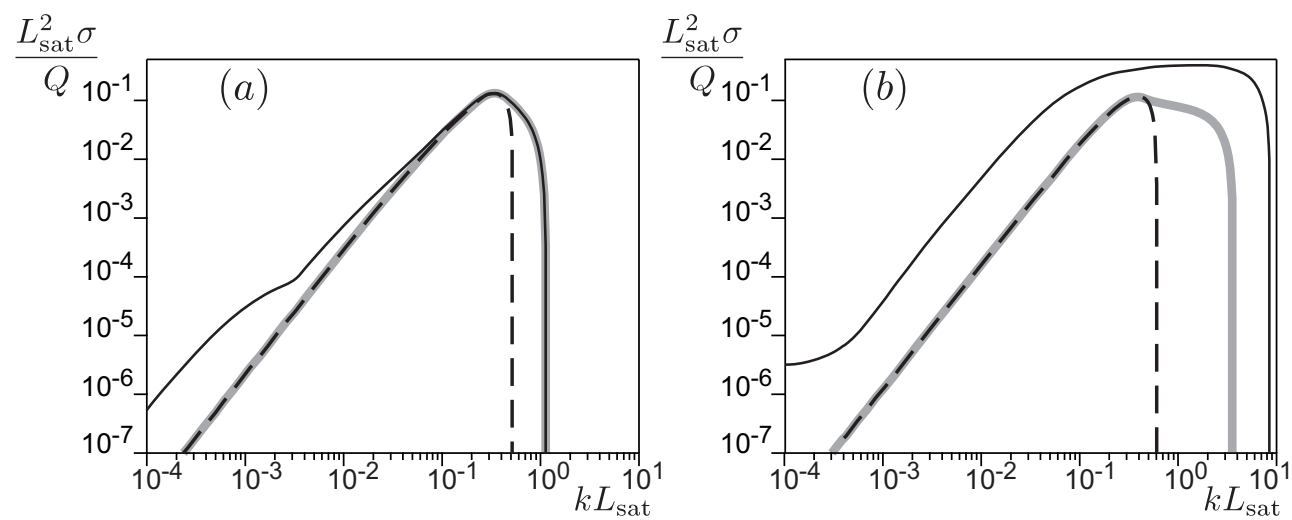

FigURE 10. Growth rate $\sigma$ as a function of the rescaled wavenumber $k L_{\text {sat }}$ for $u_{*} / u_{\text {th }} \rightarrow 1$ and $\mathcal{F} \rightarrow 0$. Comparison between the case with (solid line) and without a free surface (dashed and gray lines). The solid and gray lines correspond to the maximum over the angle $\alpha$ of the growth rate. The dashed line is for $\alpha=0$ (transverse ripples). (a) $L_{\text {sat }} / H=2^{-4}$ (b) $L_{\text {sat }} / H=4$.

$i k c=-\left(A_{x}+i B_{x}\right) Q k / L_{\mathrm{sat}}$. In the limit of large saturation length $\left(L_{\mathrm{sat}} / H \rightarrow \infty\right)$, the ratio of the water depth to the wavelength of the most unstable mode becomes small (Fig. 7a). As a consequence, one can use the shallow-water approximation and Saint-Venant's equations to describe the corresponding bedforms. Incidentally, Callander (1969) proposed his stability theory for alternate bars formation in this framework. The growth rate can then be approximated by (see OSM):

$$
\sigma \sim \frac{Q}{H L_{\mathrm{sat}}}\left[1-\left(\left(\frac{3 C \mathcal{F}}{2 k H}\right)^{2}+\left(\frac{k H}{2 C}\right)^{2}+\frac{3-\mathcal{F}^{2}}{2}\right)\left(\frac{\pi}{2}-\alpha\right)^{2}\right],
$$

where $C$ is the Chezy friction coefficient. At any value of the Froude number, the maximum growth rate is reached for $\alpha_{m}=\pi / 2$, i.e. for longitudinal bedforms, in accordance with the results of the three-dimensional theory. At any angle $\alpha$, the wavelength corresponding to the maximum growth rate is $k_{m} H=\sqrt{3} C \mathcal{F}$. By comparison with the results of the three-dimensional model investigated here, the erosive limit is thus singular and does not lead to the correct prediction at large $L_{\text {sat }} / H$. Figure 7 provides a clue to explain this discrepancy. At finite $L_{\text {sat }}$, the growth rate $\sigma$ of longitudinal patterns $(\alpha=\pi / 2)$ is strictly zero. However, for $\alpha$ slightly below $\pi / 2, \sigma$ is roughly constant (within few percents) and equal to $\frac{Q}{H L_{\mathrm{s} a t}}$ over roughly 4 decades in $k H$ : a wide range of wavenumbers - not only $k_{m}$ - are expected to grow at almost the same rate. In conclusion, regarding the incision instability in the erosive limit, the linear growth can hardly be selective.

\subsection{The role of the free surface}

We have shown that ripples and chevrons result from the same linear instability in different hydrodynamical regimes. Depending on the wavenumber $k_{\infty} H$, the free surface has an influence or not on the most unstable bedforms. One can address the questions of the sign and mechanism of this effect by comparing the dispersion relations with and without a free surface (Figure 10). For the sake of convenience, both the growth rate and the wavenumber are now rescaled by the saturation length $L_{\text {sat }}$, and the other parameters remain fixed. Below the transition between ripples and chevrons, the three curves coincide around the maximum, indicating that the influence of the free surface is negligible for ripples. On the contrary, above the transition, the growth rate is larger in 
the presence of a free surface, thus showing its destabilizing effect on inclined patterns. This result must be contrasted by the stabilising role of the free surface on transverse dunes shown by Fourrière et al. 2010: these dunes are non-linear transverse pattern of rescaled wavenumber $k H$ of order one, but in the regime $L_{\text {sat }} / H \ll 1$, i.e. below the transition. For both ripples and chevrons, the destabilising mechanism originates from pure hydrodynamics. At large $k H$, it results from the simultaneous influence of inertia and turbulent shear stress. At small $k H$, it results from the simultaneous influence of gravity induced pressure and turbulent shear stress (see OSM), even when inertia dominates.

\subsection{Effect of a slope dependent transport threshold}

At the linear order, the effect of the bed slope on the sediment transport can be represented by an apparent threshold that depends on the longitudinal slope by a factor $1+\partial_{x} Z / \mu$, where $\mu$ is the avalanche slope. On the contrary, the apparent threshold does not depend on the transverse slope at this order. The direction of the particles motion $\vec{t}$ reacts to an inclined bed in the exact opposite way: it depends on the transverse slope but not on the longitudinal slope. Taking these effects into account, the saturated flux previously expressed as (2.21) now reads

$$
\vec{q}_{\mathrm{sat}}=q_{\mathrm{sat}} \vec{t}=\Omega\left(u_{*}^{2}-u_{\mathrm{th}}^{2}\right)^{\gamma}\left(\vec{e}_{\|}-\frac{u_{\mathrm{th}}}{u_{*}} \frac{\partial_{y} Z}{\mu} \vec{e}_{\perp}\right),
$$

where $\vec{e}_{\|}$and $\vec{e}_{\perp}$ are the unit vectors parallel and perpendicular to the bedform crest respectively. Keeping the same definition of the reference flux $Q$ as before, the relation between the saturated flux and the shear stresses $\tau_{x z}$ and $\tau_{y z}$ then becomes

$$
\begin{aligned}
& \frac{\hat{q}_{\mathrm{sat}}^{x}}{Q}=\frac{\hat{\tau}_{x z}}{u_{*}^{2}}-i \mu^{-1} \frac{u_{\mathrm{th}}^{2}}{u_{*}^{2}} \cos \alpha k \hat{Z} \\
& \frac{\hat{q}_{\mathrm{sat}}^{y}}{Q}=\frac{1}{\gamma}\left(1-\frac{u_{\mathrm{th}}^{2}}{u_{*}^{2}}\right)\left(\frac{\hat{\tau}_{y z}}{u_{*}^{2}}-i \mu^{-1} \frac{u_{\mathrm{th}}}{u_{*}} \sin \alpha k \hat{Z}\right)
\end{aligned}
$$

The dispersion relation remains in the same form, but the expressions for the components of the saturated flux in, and out of phase with the topography are modified as:

$$
\begin{aligned}
a_{x}=A_{x}, & b_{x}=B_{x}-\mu^{-1} \cos \alpha \frac{u_{\mathrm{th}}^{2}}{u_{*}^{2}}, \\
a_{y}=\frac{1}{\gamma}\left(1-\frac{u_{\mathrm{th}}^{2}}{u_{*}^{2}}\right) A_{y}, \quad b_{y} & =\frac{1}{\gamma}\left(1-\frac{u_{\mathrm{th}}^{2}}{u_{*}^{2}}\right)\left(B_{y}-\mu^{-1} \sin \alpha \frac{u_{\mathrm{th}}}{u_{*}}\right) .
\end{aligned}
$$

As mentioned earlier, the details of the bifurcation diagram depend on five secondary parameters: $H / z_{0}, \mathcal{F}, u_{*} / u_{\mathrm{th}}, \mu$ and $\gamma$. Figure 11 allows us to see more precisely what we mean by 'secondary'. The introduction of the slope effect, through a finite value of $\mu$, tends to sharpen the transition (solid vs dotted line in Fig. 11). However, this influence is weakened when $u_{*} / u_{\mathrm{th}}$ is increased (that is, further away from the sediment transport threshold), as the curve relating $\alpha_{m}$ to $L_{\text {sat }} / H$ becomes smoother. The influence of $\mu$ completely disappears for asymptotically large $u_{*} / u_{\mathrm{th}}$.

In any case however, if the saturation length is much smaller than water depth, the most unstable bedforms are ripples regardless of the value of these secondary parameters. Similarly, if $L_{\text {sat }}$ is much larger than $H$, only chevrons can appear. In other words, for any set of secondary parameters, the linear instability presents a transition controlled by the dominant parameter $L_{\mathrm{sat}} / H$. Nevertheless, when $L_{\mathrm{sat}} / H$ is of order one, varying the secondary parameters can also trigger the transition from ripples to chevrons. 


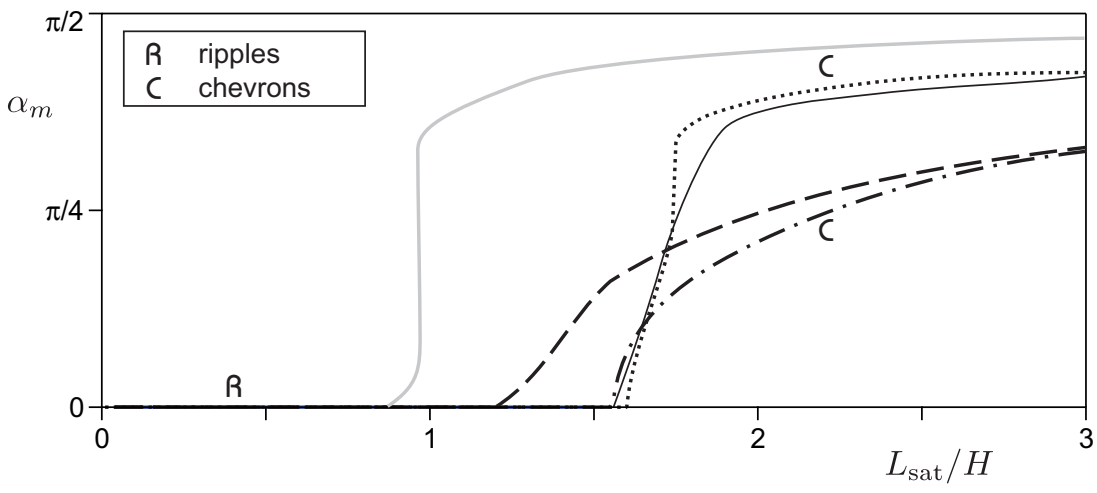

Figure 11. Pattern angle $\alpha_{m}$ of the most unstable mode as a function of $L_{\mathrm{sat}} / H$ in the limit $\mathcal{F} \rightarrow 0$. The grey solid line corresponds to the limit $u_{*} / u_{\text {th }} \rightarrow \infty$ (for any value of $\mu^{-1}$ ). The thin black solid line corresponds to the limit $\mu^{-1}=0$ and $u_{*} / u_{\mathrm{th}}=1$. The three other curves are computed with a finite avalanche slope $\mu=\tan \left(32^{\circ}\right)$, for $u_{*} / u_{\mathrm{th}}=1$ (dotted line), for $u_{*} / u_{\mathrm{th}}=2$ (dotted-dashed line) and for $u_{*} / u_{\mathrm{th}}=4$ (dashed line).

\subsection{Comparison with experiments}

How does this picture compares with experimental data? As the ratio $L_{\text {sat }} / H$ has never been considered as a control parameter before, it has not been systematically varied in experiments. In practice, small values of $L_{\text {sat }} / H$ are obtained in deep flumes, when bedload is the dominant mode of transport. On the contrary, values of $L_{\text {sat }} / H$ large enough to be in the chevron regime can be achieved in two ways. $H$ must be small if bedload is dominant, and this is typically the situation of experiments on alternate bars. Note that the flume then plays the role of a wave-guide: for a finite fixed width $W$ a relation between wavenumber $k$ and angle $\alpha$ is selected. In table 1 , we have gathered some of the experimental and field data from the literature, which show that the transitional value of $L_{\text {sat }} / H$, above which chevrons rather than ripples are observed, is around 0.1. The second possibility to get large values of $L_{\text {sat }} / H$ is to use fine particles transported in suspension by the flow. Less dense, or smaller, bed particles should then produce larger chevrons. Coming back to the aerial picture of the Loire river shown in figure 1, one can propose the following interpretation: the alternate bars form and move during flooding events, when sand grains get suspended, while the ripples and the dunes covering the sand bed form during low water, when sand grains are transported in bedload.

\section{Transition to anti-dunes}

We have previously analysed the limit of a vanishing Froude number, for which the free surface is almost flat, but still plays an important role through the pressure field. We now consider the effect of Froude number on the linear instability. We will first consider the effect of a finite Froude number in the subcritical regime $(\mathcal{F}<1)$. Then, we will show than, in the supercritical regime $(\mathcal{F}>1)$, the ratio $L_{\text {sat }} / H$ triggers the transition from ripples to anti-dunes.

\subsection{Subcritical regime}

Figure 12a shows the amplitude $\Delta$ of deformation of the free surface, rescaled by the amplitude of the bedform, for $\mathcal{F}=0.8$, in the subcritical regime. One observes that the surface is almost flat in the right part of the diagram, for $k H>1$. A transition can be observed at small wavenumbers $(k H \ll 1)$, which leads to a free surface parallel to the 


\begin{tabular}{|c|c|c|c|c|c|c|c|}
\hline References & $\begin{array}{l}H \\
(\mathrm{~m})\end{array}$ & $\begin{array}{c}d \\
(\mathrm{~mm})\end{array}$ & $\begin{array}{c}U \\
(\mathrm{~m} / \mathrm{s})\end{array}$ & $|U / \sqrt{g H}|$ & $\begin{array}{c}\mathcal{R} \\
\left(\times 10^{3}\right)\end{array}$ & $L_{\mathrm{sat}} / H$ & $\begin{array}{l}\text { transport } \\
\text { and bedforms } \\
\text { comments }\end{array}$ \\
\hline \begin{tabular}{|l} 
Ikeda (1983) \\
run 80 \\
run 102 \\
run 142
\end{tabular} & $\begin{array}{l}0.104 \\
0.012 \\
0.016\end{array}$ & $\begin{array}{c}6 \\
0.8 \\
0.8\end{array}$ & $\begin{array}{c}1 \\
0.3 \\
0.4\end{array}$ & $\begin{array}{l}1 \\
1 \\
1\end{array}$ & $\begin{array}{c}100 \\
4 \\
6\end{array}$ & $\begin{array}{l}0.6 \\
0.7 \\
0.5\end{array}$ & \begin{tabular}{|l} 
bedload \\
chevrons \\
diagonal bars \\
diagonal bars
\end{tabular} \\
\hline | Ikeda (1984) & $|0.012-0.035|$ & 1.3 & $|0.4-0.5|$ & $|0.8-1.2|$ & $5-16$ & $0.4-1$ & | bedload/bars \\
\hline $\begin{array}{l}\text { Fujita \& } \\
\text { Muramoto (1985) }\end{array}$ & $0.01-0.04$ & 1 & $|0.3-0.5|$ & | $0.8-1.2$ & $5-20$ & $0.25-1$ & \begin{tabular}{|l} 
bedload \\
alternate bars
\end{tabular} \\
\hline \begin{tabular}{|l} 
Schumm \& \\
Khan (1972)
\end{tabular} & $0.02-0.03$ & 0.7 & $|0.3-0.4|$ & $\mid$\begin{tabular}{l|l}
$0.6-0.8$
\end{tabular} & $8-9$ & $0.23-0.35$ & $\begin{array}{l}\text { bedload } \\
\text { alternate bars }\end{array}$ \\
\hline $\begin{array}{l}\text { Chang et al. } \\
(1971) \\
\text { runs 40-62 } \\
\text { runs 10-33 }\end{array}$ & $\begin{array}{l}0.02-0.08 \\
0.02-0.04\end{array}$ & $\begin{array}{l}0.2 \\
0.8\end{array}$ & $\left|\begin{array}{l}0.1-0.3 \\
0.3-0.5\end{array}\right|$ & $\begin{array}{l}0.1-0.5 \\
0.6-1.1\end{array}$ & $\begin{array}{l}5-14 \\
9-16\end{array}$ & $\begin{array}{c}10-30 \\
0.2-0.4\end{array}$ & $\begin{array}{l}\text { alternate bars } \\
\text { suspension }^{\text {(a) }} \\
\text { bedload }^{\text {(b) }}\end{array}$ \\
\hline \begin{tabular}{|l} 
Lisle et al. \\
$(1991)$ \\
$(1997)$
\end{tabular} & $\begin{array}{c}0.008 \\
0.02\end{array}$ & $\begin{array}{c}1.4 \\
0.57\end{array}$ & $\begin{array}{c}0.25 \\
0.4\end{array}$ & $\begin{array}{l}0.9 \\
0.9\end{array}$ & $\begin{array}{l}2 \\
8\end{array}$ & $\begin{array}{l}1.8 \\
0.3\end{array}$ & \begin{tabular}{|l} 
bedload \\
alternate bars \\
alternate bars
\end{tabular} \\
\hline $\mid \begin{array}{l}\text { Lanzoni } \\
(2000 \mathrm{a}) \\
(2000 \mathrm{~b})\end{array}$ & $\begin{array}{l}0.03-0.08 \\
0.04-0.07\end{array}$ & $\begin{array}{c}0.48 \\
0.2 \text { and } 2^{(\mathrm{d})}\end{array}$ & $\left|\begin{array}{l}0.3-0.5 \\
0.3-0.6\end{array}\right|$ & $\begin{array}{l}0.3-0.7 \\
0.4-0.8\end{array}$ & $\begin{array}{c}12-21 \\
20\end{array}$ & $\begin{array}{c}0.06-0.16 \\
0.03 \text { and } 0.3-0.5\end{array}$ & $\begin{array}{l}\text { bedload } \\
\text { ripples and bars }{ }^{(\mathrm{c})} \\
\text { ripples and bars }\end{array}$ \\
\hline \begin{tabular}{|l} 
Venditti \& \\
Church (2005)
\end{tabular} & 0.15 & 0.5 & $|0.4-0.5|$ & $0.3-0.4$ & $55-75$ & 0.03 & $\left|\begin{array}{l}\text { bedload } \\
\text { cross-hatch pattern }\end{array}\right|$ \\
\hline $\begin{array}{l}\text { Coleman \& } \\
\text { Melville (1996) } \\
\text { runs F2-F16 } \\
\text { runs C1-C20 }\end{array}$ & $\begin{array}{l}0.07-0.17 \\
0.08-0.17\end{array}$ & $\begin{array}{l}0.21 \\
0.83\end{array}$ & $\left|\begin{array}{c}0.4-0.7 \\
0.5-1\end{array}\right|$ & $\begin{array}{l}0.3-0.6 \\
0.4-0.9\end{array}$ & $\begin{array}{l}13-42 \\
25-50\end{array}$ & $\begin{array}{c}0.01-0.03 \\
0.05-0.1\end{array}$ & $\begin{array}{l}\text { bedload } \\
\text { ripples } \\
\text { ripples }\end{array}$ \\
\hline |Baas (1999) & 0.33 & 0.24 & $|0.3-0.6|$ & $|0.2-0.3| 1$ & $100-200 \mid$ & 0.007 & | bedload/ripples \\
\hline \begin{tabular}{|l} 
Fourrière et al. \\
$(2010)$
\end{tabular} & 0.5 & 0.33 & 0.6 & 0.3 & 300 & 0.007 & $\begin{array}{l}\text { bedload } \\
\text { ripples }\end{array}$ \\
\hline | Loire river ${ }^{(e)}$ & 4 & 0.7 & 1 & 0.15 & 4000 & 40 & | suspension $^{(\mathrm{f})} /$ bars \\
\hline
\end{tabular}

TABle 1. Some of the experimental and field data from the literature. $H$ is the flow depth. $d$ is the typical sediment size (e.g. $\left.d_{50}\right)$. $U$ is the mean velocity. $\mathcal{R}=U H / \nu$ is the flow Reynolds number. The saturation length has been computed with $L_{\text {sat }}=10 d$ for bedload (Fourrière et al. 2010) and with as $L_{\text {sat }}=H U / V_{\text {fall }}$ for transport in suspension, where $V_{\text {fall }}$ is the particle settling velocity (Claudin et al. 2011). Notes: (a,b) Transport mode is not specified in the experiment description of Chang et al. (1971). Suspension is expected in case (a) as the sediments are plastic pellets with specific gravity of 1.05. Bedload is expected for the sand grains (case b). (c) Both pattern are observed together. (d) In Lanzoni (2000b), a bimodal sediment mixture is used. Only ripples are observed when partial transport occurs, i.e. below the threshold of motion for the largest particles; Bars are observed above it. (e) These data correspond to the portion of the river displayed in figure 1c. (f) Suspension is observed during flood events. 

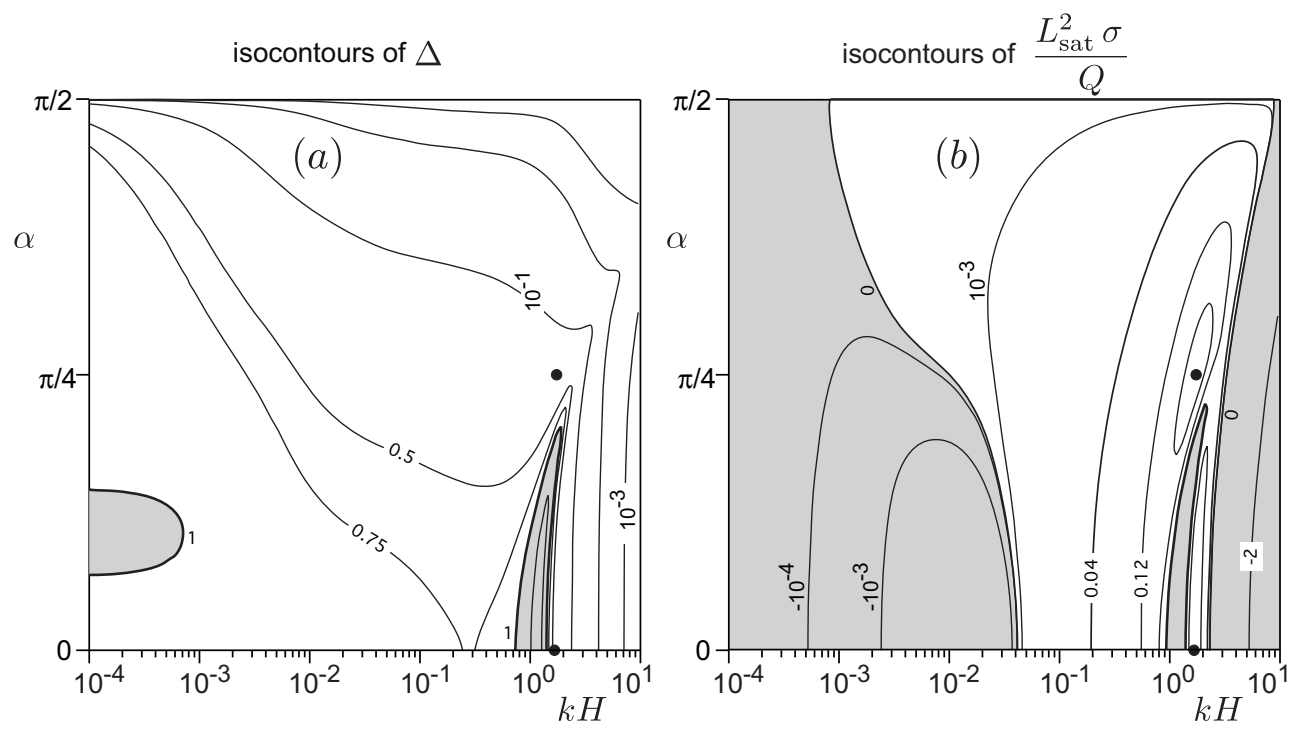

Figure 12. (a) Ratio $\Delta$ of the free surface amplitude to the pattern amplitude, as a function of the rescaled wavenumber $k H$ and of the angle $\alpha$ for $u_{*} / u_{\mathrm{th}} \rightarrow \infty$ and $\mathcal{F}=0.8$. Isocontours: $L_{\text {sat }}^{2} \sigma / Q=-10^{-4},-10^{-3},-2,0,10^{-3}, 0.04,0.12,0.16$. (b) Growth rate $\sigma$ as a function of the rescaled wavenumber $k H$ and of the angle $\alpha$ for $u_{*} / u_{\text {th }} \rightarrow \infty$ and $\mathcal{F}=0.8$. There are two local maxima of the growth rate of comparable amplitude, represented by black points $(\bullet)$. Isocontours: $L_{\text {sat }}^{2} \sigma / Q=10^{-3}, 10^{-2}, 0.1,0.5,0.75,1,2$.

bottom. Finally, there exists a sharp resonance of free surface standing waves excited by the bedforms for $k H \approx 1$. By definition, a resonance corresponds to a response maximum of an excitable system (here, the free surface) when submitted to a perturbation (here, the bedform). Resonance occurs due to the accumulation of energy when the later does not propagate i.e. when the surface wave velocity with respect to the bed vanishes. This velocity can be expressed as the difference between the flow velocity component along the direction normal to the bedform crest and the wave velocity with respect to the fluid. In the Airy linear wave theory (Phillips 1977), based on an inviscid calculation, this leads to the criterion:

$$
\mathcal{F}_{\text {res }}^{2} \simeq \frac{\tanh (k H)}{k H \cos ^{2} \alpha}
$$

Along the resonance curve, inertia and gravity-induced pressure are of the same order (see Fig. 4 for a schematic representation of the hydrodynamical regimes along the resonance curve). This results in a strong stabilising effect. Accordingly, figure $12 \mathrm{~b}$ shows that the growth rate $\sigma$ becomes negative for the values of $k H$ and $\alpha$ for which the free surface is strongly distorted.

Figure 13 shows the wavenumber and the angle for which the growth rate is maximum. At a finite Froude number, the overall picture remains similar to the case $\mathcal{F} \rightarrow 0$ : there exists a transition between ripples and chevrons controlled by the ratio $L_{\text {sat }} / H$. Looking in more details however, one can observe that the transition occurs at a smaller value of $L_{\text {sat }} / H$ for a finite $\mathcal{F}$ (a few $10^{-1}$ for $\mathcal{F}=0.8$ ). Moreover, around the transition, two maxima of comparable amplitude can coexist over a certain range of the ratio $L_{\mathrm{sat}} / H$, one located on the $\alpha=0$ axis, and the other at a finite $\alpha$. In this range, one then expects to see simultaneously ripples and chevrons (grey zone on figure 13). As a matter of fact, this phenomenon has been reported experimentally by Devauchelle et al. (2010a). 

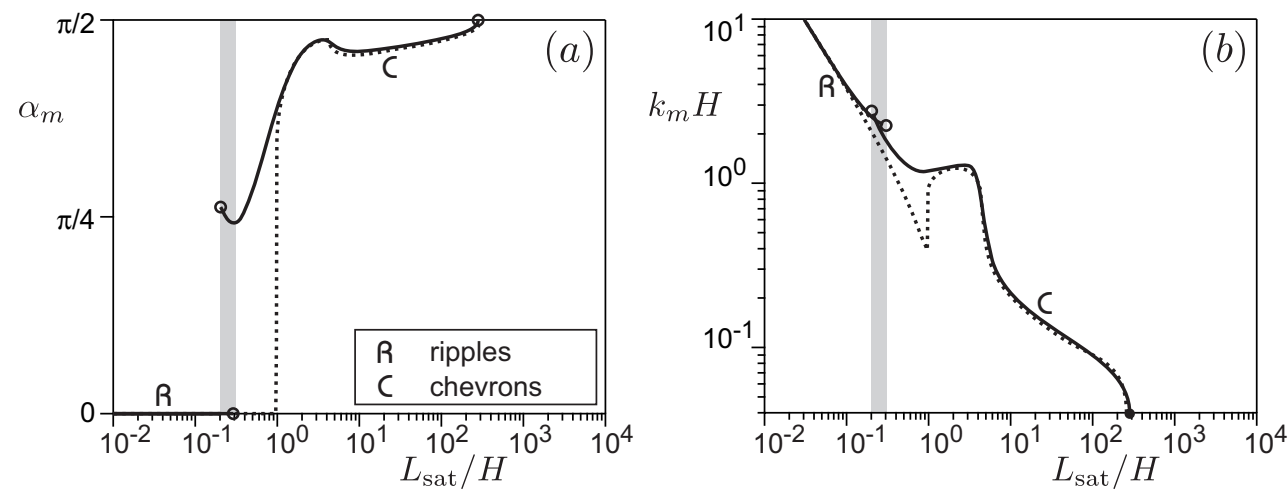

FiguRE 13. Wavelength $k_{m}$ and angle $\alpha_{m}$ of the most unstable mode as a function of $L_{\text {sat }} / H$, in the limit $u_{*} / u_{\mathrm{th}} \rightarrow \infty$. The solid line corresponds to $\mathcal{F}=0.8$ (transitions at $L_{\mathrm{sat}} / H \simeq 0.2$, at $\simeq 0.3$ and at $\simeq 2.910^{2}$ marked by $\circ$ ) and the dotted line to the limit $\mathcal{F} \rightarrow 0$ (solid line in Fig. 7). The gray zone indicates the simultaneous existence of two growth rate maxima of comparable amplitude.

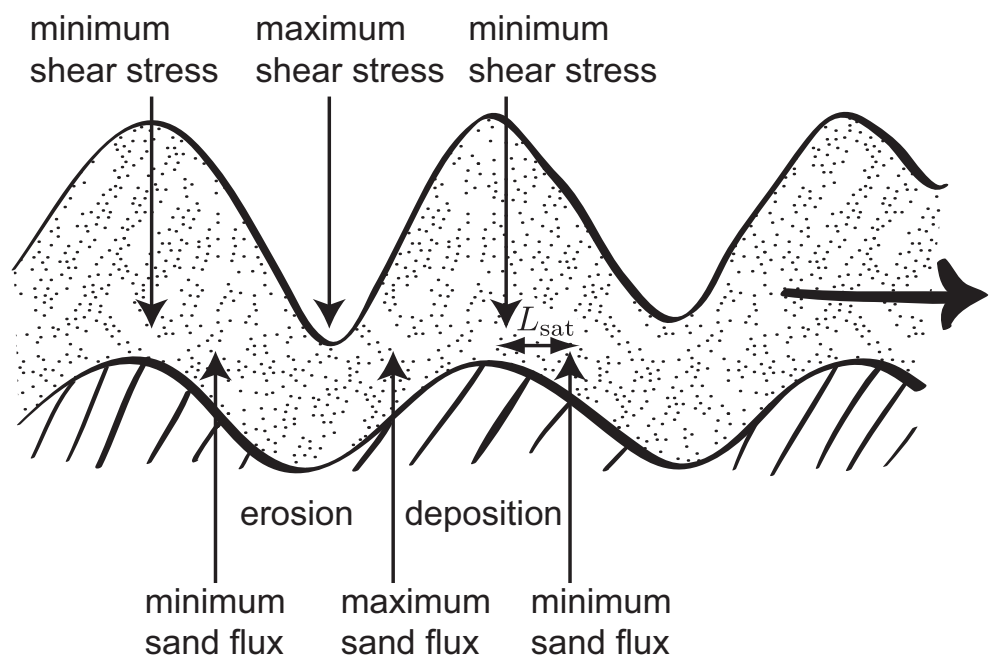

FIgURE 14. Schematic view of anti-dunes and scketch of the instability mechanism. The saturation length $L_{\text {sat }}$ represents the spatial lag between the shear stress and the sediment flux.

\subsection{Origin of anti-dunes}

Downstream propagating bedforms (ripples and chevrons) are associated with positive values of the four components $A_{x}, A_{y}, B_{x}$ and $B_{y}$. Then, the instability is due to the phase lag between the shear stress and the topography (component $B_{x}$ ) and is limited by the saturation length. In that case, the flow velocity is maximum on the crests of bedforms (positive value of $A_{x}$ ). The saturated flux globally increases on the upstream side of the structure and decreases on its downstream side. As the actual flux is spatially delayed with respect to the saturated flux, the saturation transient shifts the point at which the flux is maximum downstream of the crest. This leads to an erosion of the crests and thus stabilises the pattern.

Now consider anti-dunes. They are upstream propagating bedforms. They are associated to a negative value of the component $A_{x}$, which means that the flow velocity and 


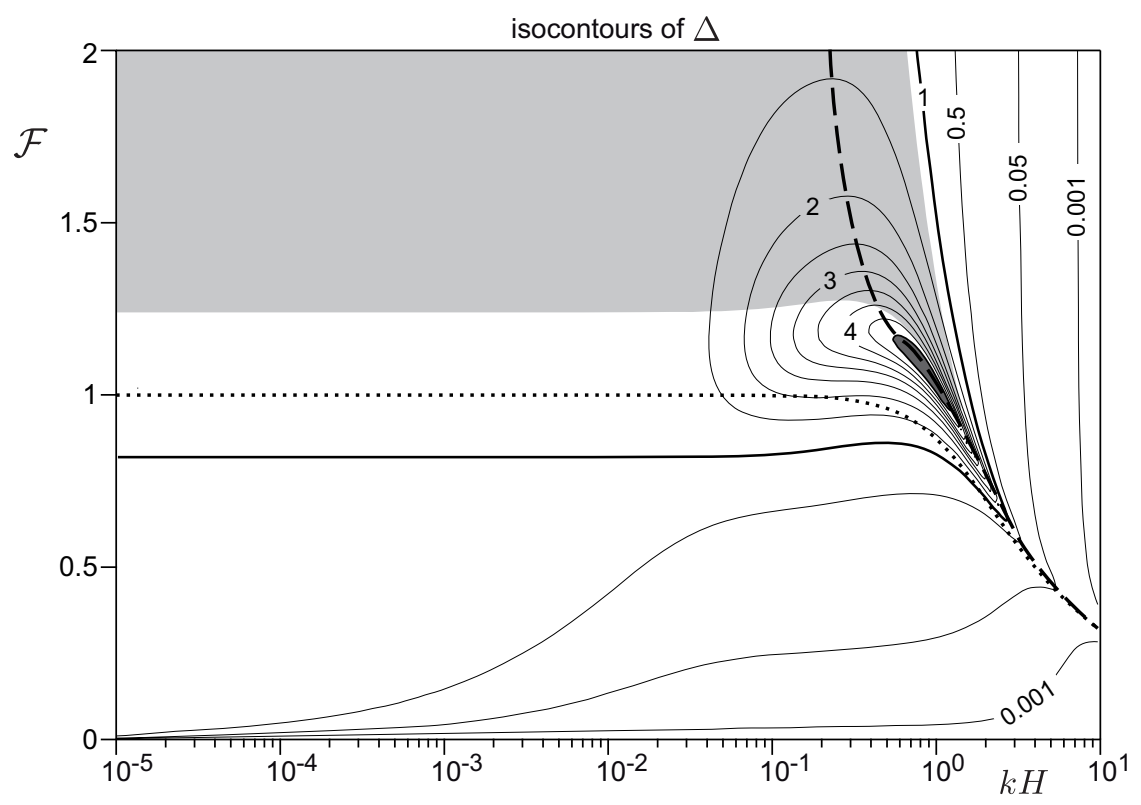

FiguRE 15. Rescaled amplitude $\Delta$ of deformation of the free surface above a transverse bedform $(\alpha=0)$ as a function of the Froude $\mathcal{F}$ and of the rescaled wavenumber $k H$. The dashed line corresponds to the resonance predicted by the inviscid equations and the dotted line to the actual resonance curve. The anti-dune region is shown in light gray. The dark gray region corresponds to strong resonance $(\Delta>5)$.

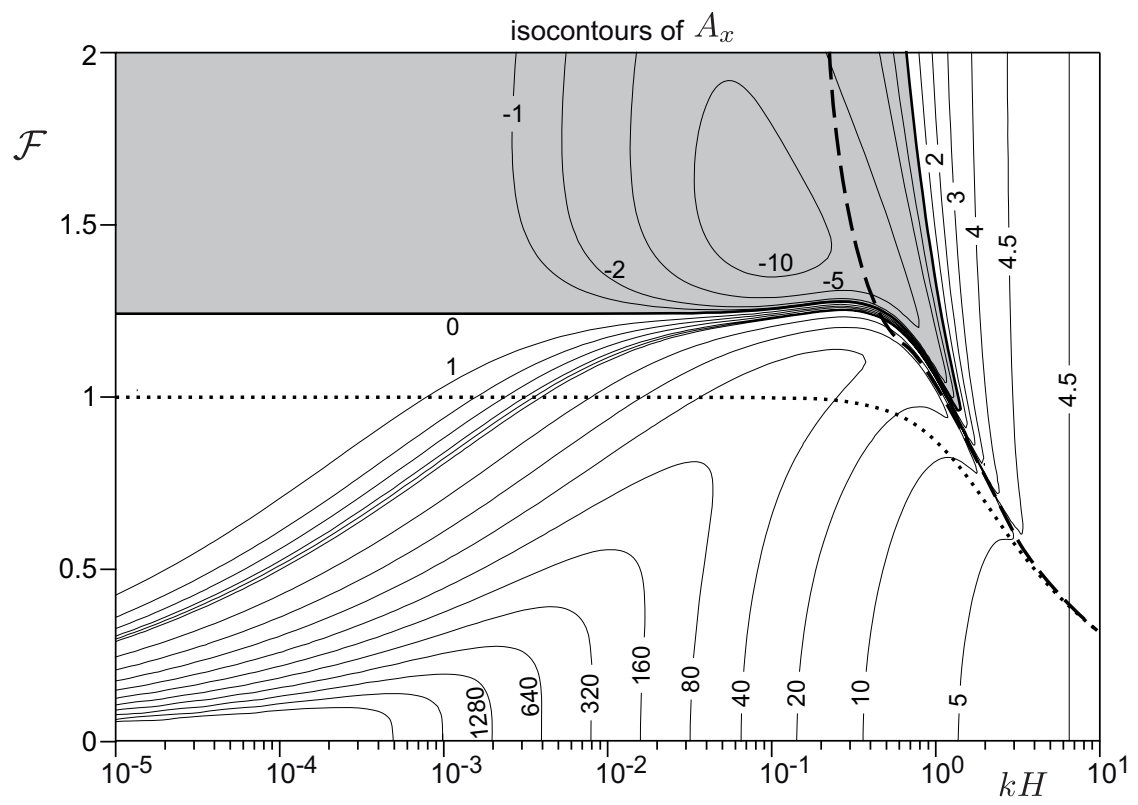

Figure 16. Component $A_{x}$ of the shear stress in phase with a transverse bedform $(\alpha=0)$ as a function of the Froude $\mathcal{F}$ and of the rescaled wavenumber $k H$. Antidunes form in the region where $A_{x}<0$ (light gray). The dotted line corresponds to the resonance predicted by the inviscid equations and the dashed line to the actual resonance curve. 

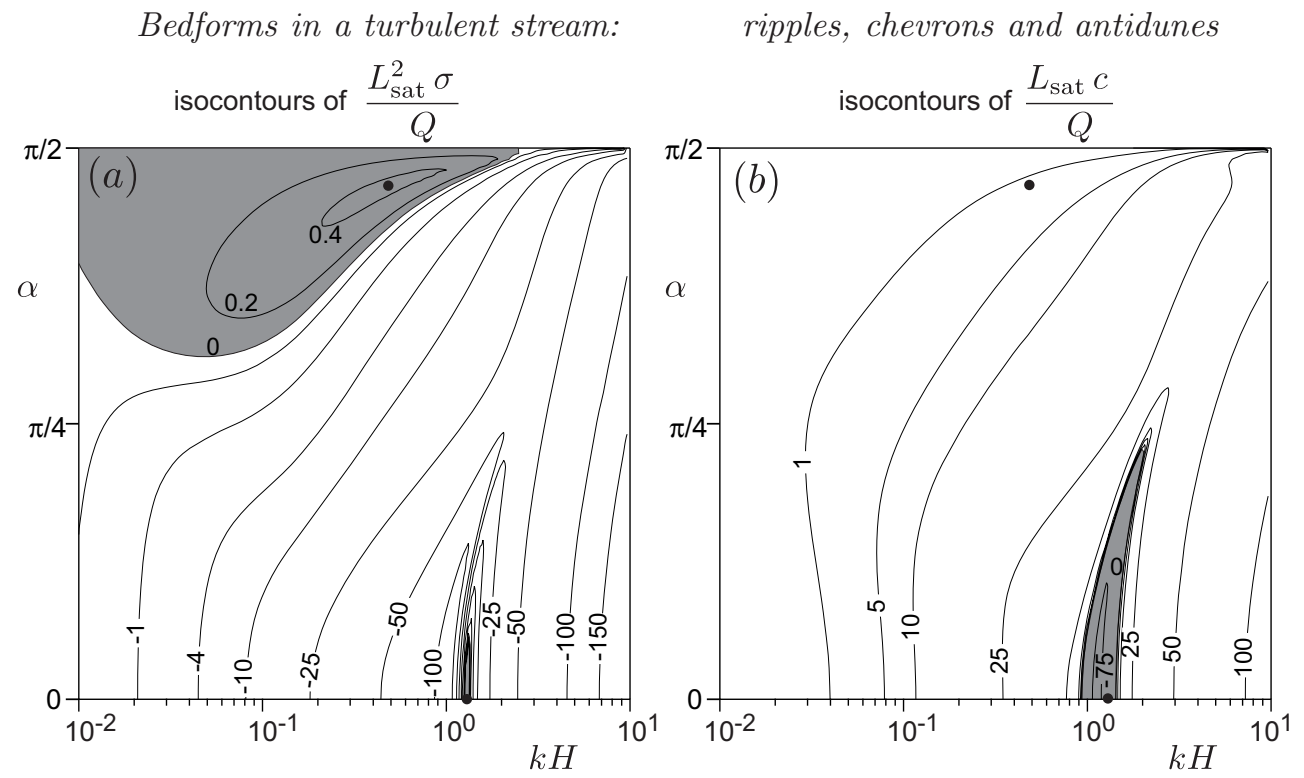

Figure 17. (a) Growth rate $\sigma$ as a function of the rescaled wavenumber $k H$ and of the angle $\alpha$ for $L_{\mathrm{sat}} / H=5, \mathcal{F}=1$ and $H / z_{0}=10^{2}$ in the limit $u_{*} / u_{\mathrm{th}} \rightarrow 1$. Isocontours for $L_{\mathrm{sat}}^{2} \sigma / Q=-150,-100,-50,-25,-10,-4,-1,0,-0.2,0.2,0.4,0.421,1.5$. Two local maxima can be observed $(\bullet)$. (b) Propagation speed $c$ in the same conditions. Isocontours for $L_{\text {sat }} c / Q=-75,0,1,5,10,25,50,100$

thus the saturated flux is minimum on their crests. The saturation transient moves the point at which the flux is minimum downstream, ensuring the crest of the anti-dune to be in the deposition zone. This means that, the delay induced by the saturation length has this time a destabilising effect. Large negative values of the shear stress component $A_{x}$ are found in the vicinity of the resonance of free surface standing waves (Figures 15 and 16).

At large Froude number, the free surface dynamics is dominated by inertia, and at low Froude number by gravity-induced pressure. In the potential flow approximation, the free surface deformation of transverse modes $(\alpha=0)$ reads

$$
\Delta=\frac{1}{\cosh (k H)-\frac{\sinh (k H)}{\mathcal{F}^{2} k H}} .
$$

$\Delta$ diverges in the resonant conditions predicted by Eq. 5.1, which is represented by dotted lines in figures 15 and 16. This formula predicts that, for $\alpha=0$, the resonant Froude number is constant, equal to 1 , at small $k H$ and decreases as $(k H)^{-1 / 2}$ for $k H$ larger than 1. The full three-dimensional model derived here, which includes turbulent stresses, leads to a picture substantially different in the supercritical regime: a resonance is observed at a Froude number larger than 1 for a rescaled wavenumber $k H$ of order 1 (Fig. 15). The above inviscid prediction is only recovered for $\mathcal{F}$ smaller than 1 or equivalently for $k H$ larger than 1 . To the best of our knowledge, this resonance curve has never been predicted nor determined experimentally, so far. It constitutes one of the most important - and surprising - results of this article.

The resonance observed in the upper part of the diagram shown in figure 15 results from different dynamical mechanisms. For a given Froude number, at asymptotically small $k H$, the flow is governed by the balance between gravity and turbulent friction: the free surface follows the bed $(\Delta=1)$. At large $k H$, the flow disturbance induced 
by the bedform decays exponentially with the distance to the bed over a typical length $k^{-1}$. The free surface amplitude is thus very small. In between, there is a small domain of wavenumber $k H$ for which the flow is governed by the balance between inertia and turbulent friction. As the influence of gravity-induced pressure is small, the free surface is then very distorted. This intermediate flow regime is precisely that for which anti-dunes are observed.

Figure 16 shows the shear stress component $A_{x}$ as a function $\mathcal{F}$ and $k H$. As expected from the previous analysis, $A_{x}$ becomes negative only at Froude number larger than 1 . Between $\mathcal{F}=1$ and $\mathcal{F} \simeq 1.25$, there is a narrow range of wavenumbers $k H$, in the vicinity of the resonant conditions, for which $A_{x}$ is negative. At larger Froude numbers, this range extends down to $k H=0$. In the next sub-section, we investigate the consequences of these features for anti-dunes.

\subsection{Supercritical regime}

Figure 17 shows a dispersion relation obtained at $\mathcal{F}=1$, in the range of parameters for which anti-dunes is the most unstable mode. One can actually see two unstable regions in the plane $(k H, \alpha)$. The upper part of the diagram corresponds to chevrons and bars, since these patterns are inclined with respect to the flow $(\alpha>0)$ and propagate in the downstream direction $(c>0)$. In the lower part of the diagram, one observes a narrow region of wavenumbers close to the resonant conditions for which the growth rate is very large and the propagation speed negative. These unstable modes lie in the region of negative $A_{x}$ and precisely correspond to anti-dunes. For the example chosen in figure 17, the growth rate is three times larger for the anti-dune mode than for the chevron mode. As previously stated, the existence of these two competing modes is due to two fundamentally different dynamical mechanisms. In particular, for ripples, chevrons and bars, the transport saturation transient is stabilising while it is destabilising for antidunes. In the later case, the range of unstable wavenumbers is controlled by the width of the free surface resonant peak, i.e. by hydrodynamics only.

From a dispersion relation like that shown in figure 17, one can extract the characteristics of the most unstable mode, which can then be plotted against the control parameters. As before, the parameter controlling the transition between the various patterns is the ratio $L_{\mathrm{sat}} / H$ of the saturation length to the flow depth (Figure 18). At small $L_{\text {sat }} / H$, the growth rate of ripples is much larger than any other mode. Therefore, the most unstable mode is associated to an angle $\alpha_{m}=0$ and a wavenumber $k_{m}$ which scales as $L_{\text {sat }}^{-1}$. The propagation speed $c_{m}$ is positive and scales as $Q / L_{\text {sat }}$; the growth rate $\sigma_{m}$ scales as $Q / L_{\text {sat }}^{2}$.

In order to understand the different transitions in figure 18, let us focus on the common behaviours and the differences between the curves obtained for $\mathcal{F}=0.9$ (dotted line) and $\mathcal{F}=1$ (dashed line). Both for $\mathcal{F}=0.9$ and $\mathcal{F}=1.0$, a first bifurcation is observed around $L_{\text {sat }} / H=0.3$, which corresponds to the ripples/inclined pattern transitions. One effectively observes that $c_{m}$ remains positive and $\alpha_{m}$ jumps from 0 to a finite value when the wavenumber $k_{m}$ crosses the resonant conditions. Above this transition, for $\mathcal{F}=0.9$, the inclined pattern remains the most unstable mode and $\alpha_{m}$ tends to $\pi / 2$ at asymptotically large $L_{\text {sat }} / H$. On the opposite, for $\mathcal{F}=1.0$, a new bifurcation takes place at $L_{\text {sat }} / H \simeq 4$. This time, the propagation speed $c_{m}$ changes sign and $k_{m}$ is selected by the resonant conditions. This bifurcation is thus associated to the transition from inclined patterns to (transverse) anti-dunes. Due to the narrow range of wavenumbers unstable, the selected wavenumber $k_{m}$ depends on $H$ only. The growth rate $\sigma_{m}$ scales asymptotically as $Q / L_{\text {sat }} H$. To summarise, the existence of anti-dunes is controlled by the Froude number $\mathcal{F}$ : below $\mathcal{F} \simeq 1, A_{x}$ is never negative. Whatever the froude number, 

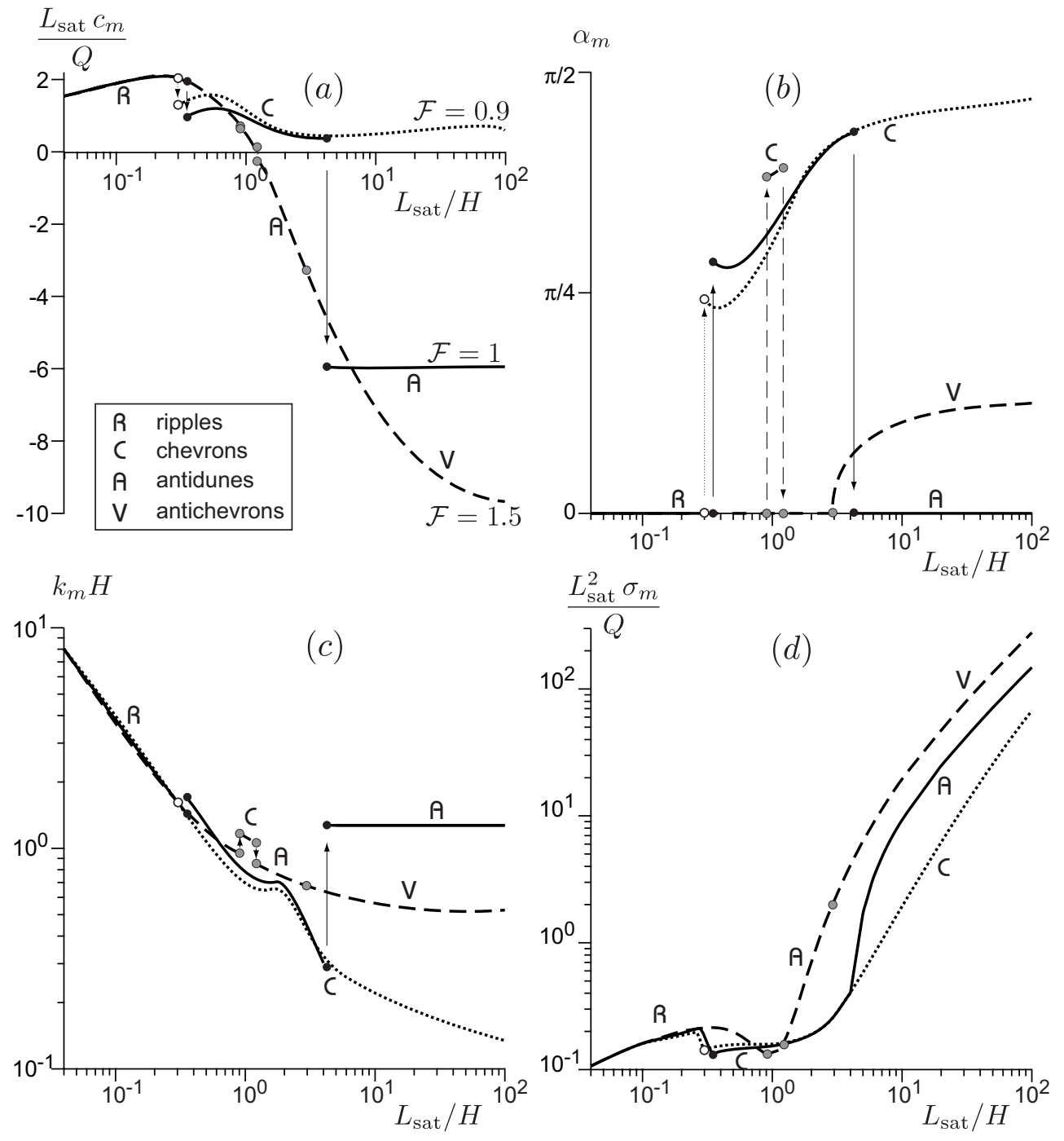

Figure 18. Characteristics of the most unstable mode as a function of $L_{\text {sat }} / H$ for three Froude numbers: $\mathcal{F}=0.9$ (dotted lines), $\mathcal{F}=1$ (solid lines) and $\mathcal{F}=1.5$ (dashed line). $H / z_{0}$ is kept constant and equal to $10^{2}$. The curves are obtained in the limit $u_{*} / u_{\text {th }} \rightarrow 1$. (a) Propagation speed $c_{m}$. Antidunes correspond to $c_{m}<0$. (b) Angle $\alpha_{m}$ of the wavenumber with respect to the flow direction. Transverse bedforms correspond to $\alpha_{m}=0$. (c) Wavenumber $k_{m}$ rescaled by the flow thickness. (d) Growth rate $\sigma_{m}$ rescaled using the saturation length $L_{\text {sat }}$ and the reference flux $Q$.

the type of bedforms that emerge is controlled by the ratio $L_{\mathrm{sat}} / H$. When this ratio is increased, for $\mathcal{F}>1$, one observes a transition from ripples to inclined patterns followed by a second transition from inclined patterns to anti-dunes.

For Froude numbers above $\mathcal{F} \simeq 1.25$, the picture becomes slightly more complicated. As shown in figure 16, $A_{x}$ is negative in a much wider range of wavenumbers going from $k H=0$ up to the resonant peak. To exemplify the consequences of this change, the characteristics of the most unstable modes are plotted in dashed line in figure 18 for $\mathcal{F}=1.5$. One observes the transition from ripples to inclined patterns at $L_{\mathrm{sat}} / H \simeq 0.92$ followed by the transition from inclined patterns to anti-dunes at $L_{\mathrm{sat}} / H \simeq 1.02$. A 


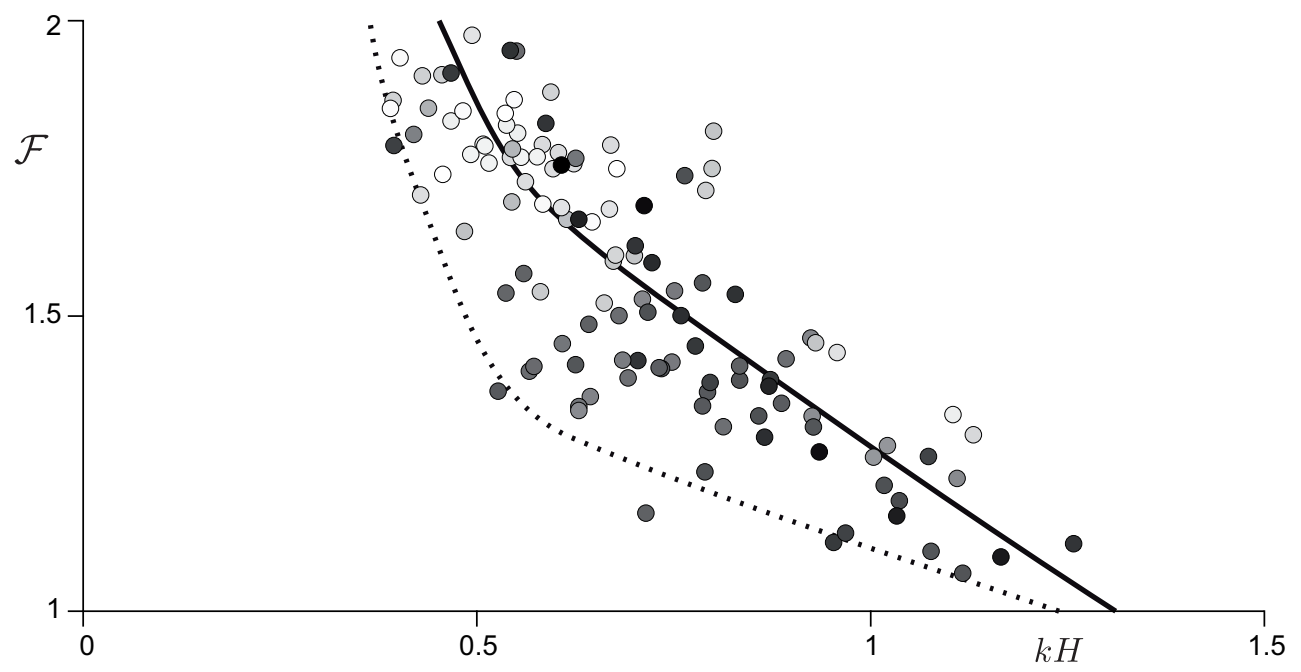

FiguRE 19. Relation between Froude number $\mathcal{F}$ and anti-dune wavenumber $k$, rescaled by the flow thickness $H$. The symbols correspond to experimental data collected by Recking et al. 2009. The gray level of the points encodes the value of $H / z_{0}$ in a logarithmic scale. White corresponds to $H / z_{0}=10^{1}$ and black to $H / z_{0}=10^{4}$. The solid line is the predicted wavenumber at the smallest value of $H / z_{0}$ for which there is an instability. The dotted line is the predicted wavenumber for $H / z_{0}=10^{2}$. In both cases, we considered the limit $u_{*} / u_{\mathrm{th}} \rightarrow 1$. The curves obtained in the other limit, $u_{*} / u_{\mathrm{th}} \rightarrow \infty$ are almost indistinguishable.

third transition, which is continuous, can be observed at $L_{\text {sat }} / H \simeq 2.95$ from antidunes transverse to the flow $\left(\alpha_{m}=0\right)$ to anti-dunes inclined with respect to the flow $\left(\alpha_{m}>0\right)$. These anti-bars or anti-chevrons have a wave-number significantly smaller than the resonant conditions. At asymptotically large $L_{\text {sat }} / H$ finite values of $k_{m} H$ and $\alpha_{m}$ are selected.

\subsection{Comparison with experiments}

We test here the predictions of the present model against the measurements available in the literature. We have used the very complete database recently gathered by Recking et al. 2009. For each data point, we have determined the ratio $H / z_{0}$ from the slope, the flow thickness and the depth average velocity, assuming that the velocity profile is logarithmic (Eq. 3.8). Then, we have built the Froude number based on the surface velocity, using again Eq. 3.8. The result is displayed in figure 19, where all data are shown in the plane $(\mathcal{F}, k H)$. Although there is some scatter in the data, a rather clear selection of the rescaled wavenumber $k H$ can be observed.

Some of the data displayed in this figure correspond to experiments where suspension is the dominant mode of sediment transport. Because we expect for this mode a saturation length $L_{\text {sat }} \propto\left(u_{*} / V_{\text {fall }}\right) H$ (Claudin et al. 2011), the ratio $L_{\text {sat }} / H$ is typically large enough to induce the formation of anti-dunes. For these data, the ratio $H / z_{0}$ is also large and they appear in dark points in the lower part of the diagram. Other data correspond to experiments for which grains are transported by bed-load. In this case, the saturation length is proportional to the grain diameter $d$ (Fourrière et al. 2010), but the flow thickness is also reduced to few grain diameters, so that $L_{\text {sat }} / H$ is still sufficiently large for anti-dunes to emerge. For these data, the ratio $H / z_{0}$ is then small. Indeed, $z_{0}$ is a fraction of grain diameter $d$. These data are displayed with light points.

The most unstable mode predicted by the model derived here mainly scales with the 
flow thickness $H$, but also depends on different subdominant parameters, most of which have not been measured experimentally. In figure 19, we focus on the influence of $H / z_{0}$. Both the solid and the dotted lines show the predictions of the model for asymptotically large $L_{\mathrm{sat}} / H$ and for a shear velocity just above the transport threshold $\left(u_{*} / u_{\mathrm{th}} \rightarrow 1\right)$ using $\mu^{-1}=0$ and $\gamma=3 / 2$. The weak dependence with respect to these parameters is exemplified in the Online Supplementary. We shall emphasise that no parameter has been tuned nor fitted: the results remain essentially the same for a different choice of the subdominant parameters $\left(u_{*} / u_{\mathrm{th}}, \mu\right.$ and $\left.\gamma\right)$.

The dotted line corresponds to the prediction obtained when $H / z_{0}$ is kept constant and equal to $10^{2}$. One observes that the dependence of the selected wavenumber $k H$ on the Froude number is correctly predicted, although it underestimates the data by approximately $30 \%$. The selected wavenumber increases when the ratio $H / z_{0}$ is decreased. However, the anti-dune instability is inhibited when this ratio is too small. The solid line in figure 19 shows the prediction when $H / z_{0}$ is kept, for all Froude numbers, at the instability threshold. The agreement with data obtained at low $H / z_{0}$ (light symbols in figure 19) is pretty good.

To conclude this section, we would like to list several difficulties raised when trying to compare more finely the model with experimental data:

- Most experimental papers report the properties of mature anti-dunes, and not the wavelength selected at the early stages of the sand bed instability. However, contrarily to ripples and dunes (Fourrière et al. 2010), the anti-dune instability is limited to a narrow band of wavenumbers (at least close to $\mathcal{F}=1$ ). Therefore, there is no possibility of significant pattern coarsening that would lead to wavenumbers much smaller than that predicted by the linear stability analysis.

- Experiments are performed in channels of finite width. As a consequence, the stream plays the role of a waveguide, with a transverse wavenumber selected by the lateral boundary conditions. This enforces a relation between the wavenumber $k$ and the angle $\alpha$ which must be taken into account in the linear stability analysis, but that have not addressed here.

- The parameters entering the description of sediment transport, namely the saturation length $L_{\mathrm{sat}}$, the ratio $u_{*} / u_{\mathrm{th}}$, the effective friction coefficient $\mu$ and the exponent $\gamma$ are difficult to measure experimentally. In the spirit of the inverse method used in Fourrière et al. 2010, these parameters could be determined if a coherent series of measurements was performed with the same grains for different $H$ and different slopes.

\section{Summary and perspectives}

We have shown that ripples and chevrons result from the same instability, although in different hydrodynamical regimes. Ripples have a wavelength smaller than the flow depth $H$ so that the flow near the bed does not feel the presence of the free surface. On the opposite, chevrons are long enough to disturb the flow over its entire depth. The flow dynamics is then controlled by the free surface. In both cases, the instability results from a phase advance of the shear stress with respect to the topography, and is limited by the saturation length $L_{\text {sat }}$. Consequently, the transition between ripples and chevrons is mainly controlled by the parameter $L_{\mathrm{sat}} / H$. This parameter reflects the ratio of the wavelength at which ripples would appear in a semi-infinite fluid to the flow thickness. When this ratio is small, the most unstable mode corresponds to transverse ripples. Conversely, chevrons and bars appear when $L_{\mathrm{sat}} / H$ is large. This suggests that alternate bars in rivers form during flooding events, when suspended load dominates over bed load. The Froude number $\mathcal{F}$ in the subcritical regime, the shear velocity ratio $u_{*} / u_{\text {th }}$ 
as well as the avalanche slope $\mu$ have a quantitative, but less crucial role. Interestingly, both ripples and chevrons are expected to coexist at the transition for $\mathcal{F}$ close to unity. Also, a pure longitudinal erosive mode (incision) is found in the limiting case $u_{*} / u_{\mathrm{th}} \rightarrow 1$ and $L_{\mathrm{sat}} / H \rightarrow \infty$.

When the Froude number is larger than 1, anti-dunes appear when $H / z_{0}$ and $L_{\text {sat }} / H$ are sufficiently large. This new mode is related to a destabilising mechanism which is different from that leading to ripples, bars and chevrons. Around resonant conditions for free surface standing waves, the flow velocity is lower on crests than on throughs. As a consequence, the sediment transport saturation transient, characterised by the saturation length $L_{\text {sat }}$, becomes destabilising. The wavelength selected by the linear instability mostly scales on the flow thickness $H$, with subdominant dependies on $\mathcal{F}, H / z_{0}$ and $L_{\text {sat }} / H$. The output of the model nicely agrees with existing experimental data. Our results also predict the emergence, at large enough Froude numbers, of 'anti-chevrons' or 'anti-bars', i.e. bedforms inclined with respect to the flow and propagating upstream, whose experimental evidence is an interesting goal.

In conclusion, field measurements and new experiments are required to explore in a continuous way the role of the parameter $L_{\text {sat }} / H$ regarding the emergence of bedforms, and the model needs to be extended toward non-linear pattern dynamics to investigate the eventual wavelength and amplitude selection at long time.

We are grateful to S. Rodrigues and A. Latapie for data on the Loire river. We thank L. Langevin for permission to use his sand pattern photo. We thank G. Festenoude for stimulating thoughts on Laplace problems. O. Devauchelle's present address is: Institut de Physique du Globe de Paris, 1 rue Jussieu, 75238 Paris Cedex 05, France. This work has benefited from the financial support of the Agence Nationale de la Recherche, grant 'Zephyr' (\#ERCS07_18).

\section{REFERENCES}

Alexander, J., Bridge, J.S., Cheel, R.J. \& Leclair, S.F. 2001 Bedforms and associated sedimentary structures formed under supercritical water flows over aggrading sand beds. Sedimentology 48, 133-152.

Andreotti, B., Claudin, P. \& Douady, S. 2002 Selection of dune shapes and velocities. Part 2: A two-dimensional modelling. Eur. Phys. J. B 28, 341-352.

Andreotti, B. \& Claudin, C. 2007 Comment on 'Minimal size of a barchan dune'. Phys. Rev. E 76, 063301.

Andreotti, B., Claudin, P. \& Pouliquen, O. 2010 Measurements of the aeolian sand transport saturation length. Geomorphology 123, 343-348.

Ashley, G.M. 1990 Classification of large scale subaqueous bedforms: a new look at an old problem. J. Sedim. Res. 60, 161-172.

Baas, J.H. 1994 A flume study on the development and equilibrium morphology of current ripples in very fine sand. Sedimentology $\mathbf{4 1}, 185-209$.

Baas, J.H. 1999 An empirical model for the development and the equilibrium morphology of current ripples in fine sand. Sedimentology 46, 123-138.

Bagnold, R.A. 1956 The flow of cohesionless grains in fluids. Phi. Trans. R. Soc. London A 249, 235-297.

Callander, R.A. 1969 Instability and river channels. J. Fluid Mech. 36, 465-480.

Carling, P.A. \& Shvidchenko, A.B. 2002 A consideration of the dune: anti-dune transition in fine gravel. Sedimentology 49, 1269-1282.

Chang, H.Y. \& Simons, D.B. 1970 The bed configuration of straight sand-bed channels when flow is nearly critical. J. Fluid Mech. 42, 491-495. 
Chang, H.Y., Simons, D.B. \& Woolhiser, D.A. 1971 Flume experiments on alternate bar formation. J. Waterways Harbors Costal Eng. Div. 97, 155-165.

Charru, F. 2006 Selection of the ripple length on a granular bed sheared by a liquid flow. Phys. Fluids 18, 121508.

Chin, A. 1999 On the origin of step-pool sequences in mountain streams. Geophys. Res. Lett. 26, 231-234.

Claudin, P., Charru, F. \& Andreotti, B. 2011 Transport relaxation time and length scales in turbulent suspensions. J. Fluid Mech. 671, 491-506.

Coleman, S.E. \& Melville, B.W. 1994 Bed-form development. J. Hydr. Engrg. 120, 544-560.

Coleman, S.E. \& Melville, B.W. 1996 Initiation of bed forms on a flat sand bed. J. Hydraul. Eng. 122, 301-310.

Coleman, S.E., Fedele, J.J. \& Garcia, M.H. 2003 Closed-conduit bed-form initiation and development. J. Hydr. Engrg. 129, 956-965.

Colombini, M. 2004 Revisiting the linear theory of sand dune formation. J. Fluid Mech. 502, $1-16$.

Colombini, M. \& Stocchino, A. 2005 Coupling or decoupling bed and flow dynamics: Fast and slow sediment waves at high Froude numbers. Phys. Fluids 17, 036602.

Colombini, M. \& Stocchino, A. 2008 Finite-amplitude river dunes. J. Fluid Mech. 611, 283-306.

Curran, J.C. 2007 Step-pool formation models and associated step spacing. Earth Surf. Proc. Landforms 32, 1611-1627.

Daerr, A., Lee, P. Lanuza, J. \& Clément, E. 2003 Erosion patterns in a sediment layer. Phys. Rev. E 67, 065201(R).

Devauchelle, O., Malverti, L., Lajeunesse, E., Josserand, C., Lagrée, P.-Y., \& Métivier, F. 2010 Rhomboid beach pattern: a laboratory investigation. J. Geophys. Res. 115, F02017.

Devauchelle, O., Malverti, L., Lajeunesse, E., Lagrée, P.-Y., Josserand, C. \& Nguyen Thu-Lam, K.-D. 2010 Stability of bedforms in laminar flows with free surface: from bars to ripples. J. Fluid Mech. 642, 329-348.

Einstein, H.A. 1950 The bed load function for sedimentation in open channel flows. Technical bulletin (US Dept. of Agriculture), 1026, 1-69.

Engelund, F. 1970 Instability of erodible beds. J. Fluid Mech. 42, 225-244.

Engelund, F. \& Fredsøe, J. 1982 Sediment ripples and dunes. Ann. Rev. Fluid Mech. 14, 13-37.

Fernandez Luque, R. \& van Beek, R. 1976 Erosion and transport of bed-load sediment. J. Hydraul. Res. 14, 127-144.

Fourrière, A., Claudin P. \& Andreotti, B. 2010 Bedforms in a turbulent stream: formation of ripples by primary linear instability and of dunes by non-linear pattern coarsening. J. Fluid Mech. 649, 287-328.

Fredsøe, J. 1978 Meandering and braiding of rivers. J. Fluid Mech. 84, 609-624.

Fujita, Y. \& Muramoto, Y. 1985 Studies on the process of development of alternate bars. Bull. Disas. Prev. Res. Inst. (Kyoto Univ.) 35, 55-86.

Gyr, A. \& Schmid, A. 1989 The different ripple formation mechanism J. Hydraul. Res. 27, 61-74.

Hayashi, T. 1970 Formation of dunes and anti-dunes in open channels. J. Hydraul. Div. 96, 357-366.

Huang, L.-H. \& Chiang, Y.-L. 2001 The formation of dunes, anti-dunes and rapidly damping waves in alluvial channels. Int. J. Numer. Anal. Meth. Geomech. 25, 675-690.

Hunt, J.C.R., Leibovich, S. \& Richards, K.J. 1988 Turbulent shear flows over low hills. Q. J. R. Meteorol. Soc. 114, 1435-1470.

Ikeda, H. 1983 Experiments on bedload transport, bedforms, and sedimentary structures using fine gravel in the 4-meter-wide flume. Environ. Res. Center pap. (Tsukuba Univ.) 2, 1-78.

Ikeda, S. 1984 Prediction of alternate bar wavelength and height. J. Hydraul. Eng. 110, 371-386.

Izumi, N. \& Parker, G. 1995 Inception of channelization and drainage basin formation: upstream-driven theory. J. Fluid Mech. 283, 341-363.

Izumi, N. \& Parker, G. 2000 Linear stability analysis of channel inception: downstream-driven theory. J. Fluid Mech. 419, 239-262.

Jackson, P.S. \& Hunt, J.C.R. 1975 Turbulent wind flow over a low hill. Q. J. R. Meteorol. Soc. $101,929$.

Julien, P.Y. 1998 Erosion and sedimentation. Cambridge University Press. 
Karcz, I. \& Kersey, D. 1980 Experimental study of free-surface flow instability and bedforms in shallow flows. Sediment. Geol. 27, 263-300.

Kennedy, J.F. 1963 The mechanics of dunes and antidunes in erodible bed channels. J. Fluid Mech. 16, 521-544.

Kostic, S., Sequeiros, O., Spinewine, B. \& Parker, G. 2010 Cyclic steps: A phenomenon of supercritical shallow flow from the high mountains to the bottom of the ocean, J. Hydroenvironment Res. 3, 167-172.

Kubo, Y. \& Yokokawa, M. 2001 Theoretical study on breaking of waves on anti-dunes, Spec. Publs. int. ass. sediment 31, 65-70.

Lajeunesse, E., Malverti, L. \& Charru, F. 2010 Bedload transport in turbulent flow at the grain scale: experiments and modeling. J. Geophys. Res. 115, F04001.

Langlois, V. \& Valance, A. 2007 Formation and evolution of current ripples on a flat sand bed under turbulent water flow. Eur. Phys. J. E 22, 201-208.

Lanzoni, S. 2000 Experiments on bar formation in a straight flume. 1. Graded sediment. Water Resources Res. 36, 3337-3349.

Lanzoni, S. 2000 Experiments on bar formation in a straight flume. 2. Uniform sediment. Water Resources Res. 36, 3351-3363.

Lenzi, M.A. 2001 Step-pool evolution in the rio Cordon, northeastern Italy. Earth Surf. Proc. Landforms 26, 991-1008.

Lisle, T.E., Ikeda, H. \& Iseya, F. 1991 Formation of stationary alternate bars in a steep channel with mixed-size sediment: a flume experiment. Earth Surf. Proc. Landforms 16, 463-469.

Lisle, T.E., Pizzuto, J.E., Ikeda, H., Iseya, F. \& Kodama, Y. 1997 Evolution of a sediment wave in an experimental channel. Water Resources Res. 33, 1971-1981.

Mantz, P.A. 1978 Bedforms produced by fine, cohesionless, granular and flakey sediments under subcritical water flows. Sedimentology 25, 83-103.

McLean, S.R. 1990 The stability of ripples and dunes. Earth-Science Rev. 29, 131-144.

Meyer-Peter, E. \& Müller, R. 1948 Formulas for bed load transport. Proc., 2nd Meeting, IAHR, Stockholm, Sweden, 39-64.

Morton, R.A. 1978 Large-scale rhomboid bed forms and sedimentary structures associated with hurricane washover. Sedimentology 25, 183-204.

Parker, G. 1975 Sediment inertia as cause of river anti-dunes. J. Hydraul. Div. 101, 211-221.

Parker, G. 1976 On the cause of characteristic scales of meandering and braiding in rivers. $J$. Fluid Mech. 76, 457-480.

Parker, G. \& Izumi, N., 2000 Purely erosional cyclic and solitary steps created by flow over a cohesive bed. J. Fluid Mech. 419, 203-238.

Phillips O.M. The dynamics of the upper ocean. Cambridge University Press, London (1977).

Raudkivi, A.J. 1966 Bedforms in alluvial channels. J. Fluid Mech. 26, 507-514.

Raudkivi, A.J. 2006 Transition from Ripples to Dunes. J. Hydr. Engrg. 132, 1316-1320.

Raudkivi, A.J. \& Witte, H.H. 1990 Development of Bed Features. J. Hydr. Engrg. 116, 10631079

Rauen, W.B., Lin, B. \& Falconer, R.A. 2008 Transition from wavelets to ripples in a laboratory flume with a diverging channel. Int. J. Sedim. Res. 23, 1-12.

Partheniades, E. Erosion and deposition of cohesive soils. J. Hydraul. Div. 91, 105-139.

Recking, A., Bacchi, V., Naaim, M., Frey, P. 2009 Antidunes on steep slopes J. Geophys. Res. 114, F04025.

Reynolds, A.J. 1965 Waves on the erodible bed of an open channel. J. Fluid Mech. 22, 113-133.

Richards, K.J. 1980 The formation of ripples and dunes on an erodible bed. J. Fluid Mech. 99, 597-618.

Robert, A. \& Uhlman, W. 2001 An experimental study of the ripple-dune transition. Earth Surf. Process. Landforms 26, 615-629.

van Rijn, L.C. 1984 Sediment pick-up functions. J. Hydr. Engrg. 110, 1494-1502.

van Rijn, L.C. 1984 Sediment transport, Part II: Suspended load transport. J. Hydr. Engrg. 110, 1613-1641.

Rouse, H. 1936 Modern conceptions of the mechanics of fluid turbulence. Trans. ASCE, paper number 1965, 463-543.

Sauermann, G., Kroy, K. \& Herrmann, H.J. 2001 Continuum saltation model for sand dunes. 
Phys. Rev. E 64, 031305.

Schumm, S.A. \& Khan, H.R. 1972 Experimental study of channel patterns. Geol. Soc. Am. Bull. 83, 1755-1770.

Summer, B. M. \& Bakioglu, M. 1984 On the formation of ripples on an erodible bed. J. Fluid Mech. 144, 177-190.

Tubino, M., Repetto, R. \& Zolezzi, G. 1999 Free bars in rivers. J. Hydraul. Res. 37, 759-775.

Vanoni, V.A. 1946 Transportation of suspended sediment by water. Trans. ASCE 111, 67-133.

Venditti, J.G., Church, M.A. \& Bennett, S.J. 2005 Bed form initiation from a flat sand bed. J. Geophys. Res. 110, F01009.

Weichert, R.B., Bezzola, G.R. \& Minor, H.-E. 2008 Bed morphology and generation of step-pool channels, Earth Surf. Proc. Landforms 33, 1678-1692.

Whittaker, J.G., \& Jaeggi, M. 1982 Origin of step-pool systems in mountain streams. J. Hydraul. Div. 108, 758-773.

Woodford, A.O. 1935 Rhomboid ripple mark. Am. J. Sci. 29, 518-525. 